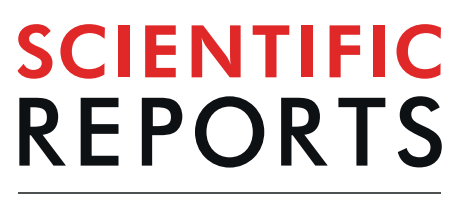

\title{
Photoinduced damage of AsLOV2 domain is accompanied by increased singlet oxygen production due to flavin dissociation
}

\begin{abstract}
Martina Petrenčákovái, ${ }^{1,}$, František Filandr ${ }^{2,6}$, Andrej Hovan ${ }^{1,6}$, Ghazaleh Yassaghi², Petr Man' ${ }^{2}$, Tibor Kožár ${ }^{3}$, Marc-Simon Schwer ${ }^{4}$, Daniel Jancura ${ }^{1,3}{ }^{3}$, Andreas Plückthun ${ }^{4}$, Petr Novák ${ }^{2}$, Pavol Miškovský3, ${ }^{3,5}$ Gregor Bánó ${ }^{1,3^{*}}$ \& Erik Sedlák ${ }^{3 *}$

Flavin mononucleotide (FMN) belongs to the group of very efficient endogenous photosensitizers producing singlet oxygen, ${ }^{1} \mathrm{O}_{2}$, but with limited ability to be targeted. On the other hand, in geneticallyencoded photosensitizers, which can be targeted by means of various tags, the efficiency of FMN to produce ${ }^{1} \mathrm{O}_{2}$ is significantly diminished due to its interactions with surrounding amino acid residues. Recently, an increase of ${ }^{1} \mathrm{O}_{2}$ production yield by $\mathrm{FMN}$ buried in a protein matrix was achieved by a decrease of quenching of the cofactor excited states by weakening of the protein-FMN interactions while still forming a complex. Here, we suggest an alternative approach which relies on the blue light irradiation-induced dissociation of FMN to solvent. This dissociation unlocks the full capacity of FMN as ${ }^{1} \mathrm{O}_{2}$ producer. Our suggestion is based on the study of an irradiation effect on two variants of the LOV2 domain from Avena sativa; wild type, AsLOV2 wt, and the variant with a replaced cysteine residue, AsLOV2 C450A. We detected irradiation-induced conformational changes as well as oxidation of several amino acids in both AsLOV2 variants. Detailed analysis of these observations indicates that irradiationinduced increase in ${ }^{1} \mathrm{O}_{2}$ production is caused by a release of $\mathrm{FMN}$ from the protein. Moreover, an increased FMN dissociation from AsLOV2 wt in comparison with AsLOV2 C450A points to a role of C450 oxidation in repelling the cofactor from the protein.
\end{abstract}

Flavin mononucleotide (FMN) belongs to a group of efficient endogenous photosensitizers in cells with rather high singlet oxygen, ${ }^{1} \mathrm{O}_{2}$, quantum yield $\left(\Phi_{\Delta}\right)$ within the range $0.51-0.65^{1,2}$. Depending on FMN concentrations and concentrations of available oxygen, the flavin(s) can be even more effective ${ }^{1} \mathrm{O}_{2}$ generators than exogenous porphyrins used for cell killing in photodynamic therapy (PDT). To minimize the potential deleterious effect of flavins to cells, the isoalloxazine moiety of flavin cofactors is typically deeply buried in the protein core of flavoenzymes $^{3}$ or storage proteins ${ }^{4}$.

Singlet oxygen, the lowest energy excited electronic state of molecular oxygen, belongs to the group of reactive oxygen species (ROS), which includes superoxide anion $\left(\mathrm{O}_{2}{ }^{-}\right)$, hydrogen peroxide $\left(\mathrm{H}_{2} \mathrm{O}_{2}\right)$, and hydroxyl radi$\mathrm{cal}(\mathrm{HO})^{*}$, enabling to oxidize and/or oxygenate many biologically relevant molecules ${ }^{5,6}$. Singlet oxygen can be produced in a variety of ways by physical mechanisms, including energy transfer from the excited triplet states of particular chromophores to molecular oxygen ${ }^{7}$, or by chemical mechanisms as one of the products of peroxidase enzymes ${ }^{8}$. In biological systems, ${ }^{1} \mathrm{O}_{2}$ is usually generated by electronic energy transfer from an excited state of a photosensitive molecule, so-called photosensitizer (PS), to ground state $\mathrm{O}_{2}{ }^{6}$. The high reactivity of singlet oxygen towards biological molecules is relevant in the context of $\mathrm{PDT}^{9}$ and chromophore-assisted laser inactivation (CALI) of proteins and cells ${ }^{10,11}$.

${ }^{1}$ Department of Biophysics, Faculty of Science, P.J. Šafárik University, Jesenná 5, 041 54, Košice, Slovakia. ${ }^{2}$ BioCeV Institute of Microbiology, Průmyslová 595, 252 50, Vestec, Czech Republic. ${ }^{3}$ Center for Interdisciplinary Biosciences, Technology and Innovation Park, P.J. Šafárik University, Jesenná 5, 041 54, Košice, Slovakia. ${ }^{4}$ Department of Biochemistry, University of Zürich, Winterthurerstrasse 190, CH-, 8057, Zurich, Switzerland. ${ }^{5}$ SAFTRA photonics Ltd, Jesenná 5, 041 54, Košice, Slovakia. ${ }^{6}$ These authors contributed equally: Martina Petrenčáková, František Filandr, and Andrej Hovan. *email: gregor.bano@upjs.sk; erik.sedlak@upjs.sk 
Studies addressing the behavior and action of ${ }^{1} \mathrm{O}_{2}$ have been performed for decades, but there is still a limited knowledge on the spatially- and temporally-dependent ${ }^{1} \mathrm{O}_{2}$-induced cell signaling processes ${ }^{12,13}$. An encapsulation of a photosensitizer in a protein matrix, thus forming a genetically-encoded photosensitizer, facilitates ROS production with (i) molecular level spatial control via protein targeting, and (ii) temporal and dose control by the incident light $\mathrm{t}^{2,13,14}$.

On the other hand, the improved control regarding ${ }^{1} \mathrm{O}_{2}$ production by using genetically-encoded photosensitizers leads to attempts to utilize them as antimicrobial agents ${ }^{15,16}$ or in $\mathrm{PDT}^{17}$. Up to now, more than 400 individual compounds have been recognized as possible candidates for use as PSs ${ }^{9}$. However, a large fraction of the small organic PSs has unsuitable physico-chemical properties such as low solubility and stability in aqueous solvents, and inherent low specificity for targeted diseased tissue. Consequently, such compounds exhibit a certain toxicity to other, healthy tissues ${ }^{18,19}$. Alternatively, the use of proteins as genetically encoded ${ }^{1} \mathrm{O}_{2}$ generators offers new ways of designing, synthesizing, and targeting of biomacromolecules containing PS $^{20}$.

Recently, significant efforts have been invested into the design of protein PSs containing FMN due to its high value of $\Phi_{\Delta}{ }^{15,21-23}$. On the other hand, genetically encoded fluorescent tags have inherently very low efficiency of ${ }^{1} \mathrm{O}_{2}$ production $\left(\Phi_{\Delta}<0.09\right)^{15,20,24,25}$. These observations point to a strong effect of the surrounding protein matrix on ${ }^{1} \mathrm{O}_{2}$ production efficiency by the chromophore ${ }^{21}$.

Two major ways how the protein environment diminishes the yield of ${ }^{1} \mathrm{O}_{2}$ production have been identified: (i) inefficient diffusion of molecular oxygen through the protein scaffold to the site of PS localization and (ii) quenching of the excited state of PS, e.g. FMN triplet state, by the protein environment ${ }^{23}$. In fact, efficiency of the ${ }^{1} \mathrm{O}_{2}$ production in miniSOG (mini-singlet oxygen generator; $\Phi_{\Delta}=0.03-0.05$ ) ${ }^{21,26}$, engineered from the FMN-containing LOV2 domain of Arabidopsis thaliana phototropin $2^{27}$, upon chemical denaturation increased over 10 -fold in comparison with its native form ${ }^{21}$. Consequently, these observations led to efforts to develop protein PSs with improved ${ }^{1} \mathrm{O}_{2}$ production, such as SOPP (singlet oxygen photosensitizing protein; $\left.\Phi_{\Delta}=0.19-0.26\right)^{26}$ and particularly SOPP 3 with $\Phi_{\Delta}=0.60$, comparable to that of free $\mathrm{FMN}^{23}$. These improved variants of miniSOG were obtained by identification and replacement of amino acids responsible for: (i) steric barriers for oxygen diffusion toward the PS, (ii) quenching of FMN triplet state by electron transfer, and (iii) quenching of produced ${ }^{1} \mathrm{O}_{2}$ by chemical reactions ${ }^{23,26}$.

In line with these observations is a finding of $\sim 10$-fold increase of the $\Phi_{\Delta}$ value in miniSOG after its irradia$\operatorname{tion}^{23}$. This finding was explained as the result of progressive photoinactivation of certain amino acids, such as His, Met, Phe, Trp, and Tyr, that are responsible for ${ }^{1} \mathrm{O}_{2}$ quenching ${ }^{5,8,28}$ and/or the buildup of FMN photoproducts $^{21}$. Indeed, very recently Torra et al. ${ }^{29}$ showed that the irradiation of miniSOG leads not only to oxidation of several residues, which are possible electron donors to FMN, but also to phototransformation of FMN to lumichrome, which results in facilitation of the access of molecular oxygen to the isoalloxazine ring of FMN.

In this work, we present detailed analysis of cumulative irradiation of two forms of LOV2 domain of phototropin 1 from Avena sativa (AsLOV2), wild type (wt) and its variant with replaced cysteine 450 (the numbering correspond to the original sequence of the LOV domain in phototropin 1) for alanine (C450A). These two variants differ by an ability of light-induced conformational change, which is induced by covalent bond forming between the thiol group of $\mathrm{C} 450$ and $\mathrm{C}(4 \mathrm{a})$ on the isoalloxazine ring. While AsLOV2 is able of photoswitching, the variant $\mathrm{C} 450 \mathrm{~A}$, due to removing of the thiol, has lost this property. The AsLOV2 primary structure is more than $80 \%$ identical with miniSOG. Our results clearly show different kinetics of FMN triplet states of the AsLOV2 and its variant, increased efficiency of ${ }^{1} \mathrm{O}_{2}$ production as a function of irradiation time as well as oxidative modification of both proteins. Based on these observations, we conclude that the irradiation-induced increase of ${ }^{1} \mathrm{O}_{2}$ production in the AsLOV2 variants is due to a release of FMN to solvent as a result of oxidative modification of certain amino acids in the AsLOV2 structure. Our results suggest a new approach towards designing an efficient protein photosensitizer as a carrier of a chromophore that can be subsequently released by irradiation of the protein at the site of its action.

\section{Experimental methods}

Cloning, expression and purification of the AsLOV2 domain. Wild type AsLOV2 as well as variant AsLOV2-C450A were expressed in E. coli strain BL21(DE3). The bacterial cells were grown at $37^{\circ} \mathrm{C}$ in ampicillin containing $(100 \mu \mathrm{g} / \mathrm{ml}) \mathrm{TB}$ medium until they reached $\mathrm{OD}_{600} \sim 0.6-0.8$. Protein expression was induced by adding isopropyl $\beta$-D-galactopyranoside $\left(100 \mu \mathrm{M}\right.$ final concentration) following a temperature downshift from $37^{\circ} \mathrm{C}$ to $25^{\circ} \mathrm{C}$. Expression was carried out in darkness overnight at $25^{\circ} \mathrm{C}$. The proteins were purified using metal ion affinity chromatography (Ni-NTA Superflow, Qiagen). The sequence of the final construct of AsLOV2 contains an N-terminal His Ho $_{10}$-tag, followed by amino acids 404-547 of AsLOV2 according the original numbering of phototropin 1. After IMAC purification they were run on a Superdex 75 Increase, 10/300 GL, size exclusion column and concentrated in $20 \mathrm{mM}$ TrisHCl buffer, $\mathrm{pH}$ 7.8. All steps were performed in darkness. The ratio of absorbance at $280 \mathrm{~nm} / 477 \mathrm{~nm}$ of the final protein was $\sim 2.5$, suggesting the absence of AsLOV2 apoform ${ }^{30}$.

Sample irradiation (by laser) and singlet oxygen phosphorescence. Samples (1.2 ml) containing $25 \mu \mathrm{M}$ protein were placed in a $10 \times 10 \times 40 \mathrm{~mm}$ quartz cuvette equipped with an overhead-type glass stirrer and kept at $\sim 30^{\circ} \mathrm{C}$. A laser system consisting of a pulsed optical parametric oscillator (OPO) (GWU basiScan-M) pumped with the third harmonic of a Nd:YAG laser (Spectra-Physics, Quanta-Ray, INDI-HG-10S) was used to excite the samples. The OPO wavelength was tuned to $475 \mathrm{~nm}$ matching the absorption maximum of the AsLOV2 protein. The repetition rate of the 5-7 ns long laser pulses was set to $10 \mathrm{~Hz}$. The $3 \mathrm{~mm}$ diameter laser beam was focused to the cuvette by means of a $200 \mathrm{~mm}$ lens. The average laser power on the sample was $0.9 \mathrm{~mW}$. The phosphorescence signal of singlet oxygen passed through a $1250-1300 \mathrm{~nm}$ band-pass filter and was detected with a photomultiplier tube (Hamamatsu H10330A-75) operated in photon counting mode. A multichannel scaler PCI card (Becker \& Hickl, MSA-300) was used to acquire the phosphorescence time course. In order to suppress the 
background signal originating from the optical components, the emission signal was measured with two additional band-pass filters, in the $1200-1250 \mathrm{~nm}$ and the $1300-1350 \mathrm{~nm}$ spectral regions. The background signal was assumed to have a slowly varying wavelength dependence in the covered spectral range. The resulting singlet oxygen phosphorescence was calculated by subtracting the average of the two auxiliary measurements (acquired in the adjacent spectral regions) from the signal measured in the 1250-1300 nm spectral range. The background was efficiently suppressed this way. Throughout the experiments an average of 2500 laser pulses was detected with each filter consecutively. The time needed for a single measurement set (three band-pass filters) was $12.5 \mathrm{~min}$. The total irradiation time was $75 \mathrm{~min}$.

Measurements of FMN triplet state lifetime. An additional $633 \mathrm{~nm} \mathrm{cw}$ laser was added to the optical setup to monitor the FMN triplet state lifetime in a flash-photolysis experiment ${ }^{31}$. The polarization of the $\mathrm{cw}$ laser was oriented at the magic angle with respect to the excitation beam polarization. The laser beam was passing through the sample area excited with the pulsed laser. The time-resolved absorption at $633 \mathrm{~nm}$ was measured with an avalanche photodiode (Thorlabs, APD110A2) connected to a digitizing oscilloscope (Tektronix, DPO 7254). The average signal of 2500 laser pulses was acquired consecutively throughout the irradiation experiment. Eighteen decay curves were measured during the 75 minutes interval. The protein concentration was $25 \mu \mathrm{M}$.

Determination of a light-induced released of FMN. Relative amounts of released FMN from AsLOV2 wt and AsLOV C450A were determined by FMN fluorescence after filtration using $10 \mathrm{kDa}$ cut-off filter tubes. Each sample, i.e. non-illuminated and illuminated AsLOV2 wt and AsLOV2 C450A, $900 \mu \mathrm{l}$ of $10 \mu \mathrm{M}$ protein, was loaded into Amicon Ultra Centrifugal filter tube and centrifuged for $5 \mathrm{~min}$ at $7500 \mathrm{~g}$. After the spin, the collected flow-through of each sample was measured for FMN fluorescence.

Spectral analysis. Different spectroscopic techniques have been used to follow the structural changes of AsLOV2 wild type and variant C450A after irradiation with blue light. All spectra were recorded at room temperature.

Ultraviolet and visible absorption spectra were obtained with a UV-2401PC UV-Vis spectrophotometer (Shimadzu). Protein concentrations were calculated by using an extinction coefficient of $\varepsilon_{447}=13800 \mathrm{M}^{-1} \cdot \mathrm{cm}^{-1}$ for oxidized $\mathrm{FMN}^{32}$. The measurements were performed in quartz cuvettes with $1 \mathrm{~cm}$ pathlength. The protein concentration was $25 \mu \mathrm{M}$.

Fluorescence emission spectra were recorded with a RF-5301PC spectrofluorophoto-meter (Shimadzu). The emission spectra of FMN and tryptophan were measured by using excitation wavelengths at $445 \mathrm{~nm}$ and $295 \mathrm{~nm}$, respectively. For obtaining fluorescence spectra, a protein concentration of $10 \mu \mathrm{M}$ was used.

Circular dichroism spectra measurements were performed by Jasco 810 (Jasco). The protein concentration used in CD measurements was $10 \mu \mathrm{M}$. The measurements in the far-UV and the near-UV spectral regions were performed in quartz cuvette with $1 \mathrm{~mm}$ and $1 \mathrm{~cm}$ pathlengths, respectively.

Adduct decay kinetics measurements. For light-induced adduct formation accompanied by absorbance changes in the spectral region $425-520 \mathrm{~nm}$, we used a photographic flash (Canon) as a light source. Adduct decay kinetics were measured by following absorbance at $447 \mathrm{~nm}$ in quartz cuvettes with $1 \mathrm{~cm}$ pathlength. A UV-2401PC UV-Vis recording spectrophotometer (Shimadzu) was used. In all measurements, the protein concentration was $25 \mu \mathrm{M}$.

Molecular modeling. The Maestro/Desmond ${ }^{33,34}$ combination of programs has been used for model building, energy minimization, MD simulations and analysis of simulation results. The OPLS-2005 force field (the up-to-date version of the OPLS force field family ${ }^{35-42}$ ) was used to carry out the simulation studies. The two AsLOV2 protein structures (PDB ID: 2v0u and 2v0w) were downloaded from the Protein Data Bank, updated in Maestro using the "Protein Preparation Wizard". The proteins were solvated (SPC water model ${ }^{43}$; the water molecules were added within $1 \mathrm{~nm}$ buffer around the protein, creating thus a $6 \times 6 \times 7 \mathrm{~nm}$ sized solvent box) and the resulting structures were then minimized. The final structures were equilibrated and submitted for $5 \mathrm{~ns}$ NPT (pressure at $1.01325 \mathrm{bar}$ ) simulations with the Desmond program at $300 \mathrm{~K}$. Molecular geometries resulting from simulations were saved at 0.5 ps intervals and were visualized within the Maestro and $\mathrm{VMD}^{44}$ programs and were used for further analysis. The cystine thiol of C450 of AsLOV wt (PDB ID: 2v0u) was oxidized to SOO ${ }^{-}$and the resulting structures were also solvated, and NPT simulated for $5 \mathrm{~ns}$ in Desmond as described above. Comparative simulations were then performed for the C450A-mutated AsLOV2 domain.

The Caver 3.0 program $^{45}$ has been used for analysis of possible transport tunnels in all protein structures downloaded from the RCSB protein database as well as all structures saved from MD simulations.

The BIOVIA Discovery Studio ${ }^{46}$ visualizer was also used for complementary visualization and analysis of the modeled molecular structures.

Top-down mass spectrometry. Proteins were desalted off-line on a Protein OptiTrap (Optimize Technologies, Oregon City, USA) with $0.1 \%$ formic acid (FA) in water and eluted with $80 \%$ acetonitrile $/ 0.1 \%$ FA. Protein concentration were adjusted to $5 \mu \mathrm{M}$ with water and the proteins were loaded into a quartz capillary ESI tip and mounted onto a home-built nESI source. Data were acquired in a broad band mode (m/z 200-3000) or in a CASI mode (Continuous Acquisition of Selected Ions) where selected charged states $(19+, 20+, 21+$ and $22+$ ) of the protein were analyzed and fragmented simultaneously. Protein fragmentation was done through collision-induced dissociation in the quadrupole (front end) of 15 T FT-ICR MS (solariX XR, Bruker Daltonics, Bremen, Germany). Data were interpreted by a software tool developed in the laboratory and validated manually in Data Analysis 4.1 (Bruker Daltonics). 


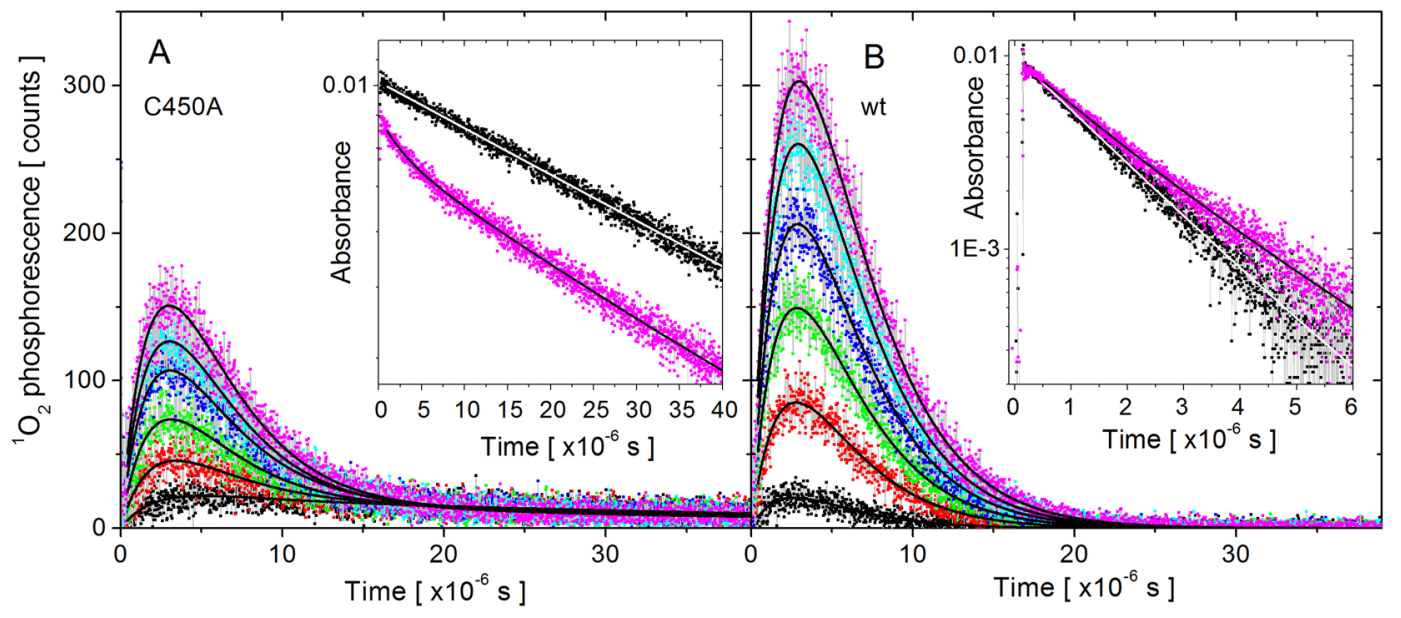

Figure 1. The time-course of the singlet oxygen phosphorescence signal for AsLOV2 C450A (A) and AsLOV2 wt (B) at different irradiation levels. The six curves were fitted in a global fitting procedure; the lines represent the fitted results. Insets: The decay of the FMN triplet state absorbance at $633 \mathrm{~nm}$ following the excitation laser pulse as measured for AsLOV2 C450A and AsLOV2 wt. Black and magenta points correspond to low and high irradiation, respectively.

Bottom-up mass spectrometry. Samples $(10 \mu \mathrm{g})$ of each wild-type and C450A AsLOV2, both irradiated and non-irradiated, were digested using trypsin or Asp-N in $100 \mathrm{mM}$ Tris- $\mathrm{HCl} \mathrm{pH} 8.5$ at $37^{\circ} \mathrm{C}$ overnight. Subsequently, the samples were analyzed using LC-MS/MS. Peptides were injected on a reversed phase trap column (Zorbax 300SB-C18 $5 \mu \mathrm{m}, 0.3 \times 5 \mathrm{~mm}$, Agilent Technologies, USA) and desalted by $0.1 \%$ FA in water for $3 \mathrm{~min}$ at flow rate of $20 \mu \mathrm{L} / \mathrm{min}$. Next, the peptides were eluted and separated on an analytical column (ZORBAX 300 SB-C18, $0.3 \times 150 \mathrm{~mm}, 3.5 \mu \mathrm{m}$, Agilent, USA) using a linear acetonitrile gradient from 5 to $35 \%$ of solvent B. Solvents were as follows: A: $0.1 \%$ FA in water, B: $0.1 \%$ FA, $95 \%$ ACN in water. The flow on the analytical column was $10 \mu \mathrm{L} / \mathrm{min}$ and the temperature was held at $50^{\circ} \mathrm{C}$. Eluting peptides were analyzed online with ESI-FT-ICR MS (15 T solariX XR, Bruker Daltonics, Germany) operating in data-dependent mode. The data were processed by DataAnalysis 4.1 (Bruker Daltonics, USA) and then searched by MASCOT in ProteinScape 4 (Bruker Daltonics, USA) against a database containing the AsLOV2 wt and C450A sequences. Various oxidative modifications of Met, Phe, His, Trp, Tyr, Pro and Cys were set as a variable. Parallel analysis in PEAKS X Studio was also conducted with automatic search for possible modifications present in the Unimod database. All found oxidized peptides and their non-oxidized variants were then manually searched in the data and their extracted ion chromatograms and highest intensity monoisotopic peaks were compared to estimate the level of oxidation. Assignment of oxidative modification to specific amino acids was done based on the fragment ions in the MS/MS spectra.

\section{Results}

In this work, singlet oxygen is generated through the interaction of triplet state FMN $\left({ }^{3} \mathrm{FMN}\right)$ with molecular oxygen. The kinetics of the ${ }^{3} \mathrm{FMN}$ transient absorption signals and ${ }^{1} \mathrm{O}_{2}$ phosphorescence were analyzed considering two assumptions. First, the small size of the studied AsLOV2 proteins ( $\sim 3.5 \mathrm{~nm}$ diameter) suggests that ${ }^{1} \mathrm{O}_{2}$ diffuses out of the protein matrix relatively fast. Due to this fact, ${ }^{1} \mathrm{O}_{2}$ spends most of its lifetime in the water environment. The justification of this assumption is given in the Supporting information.

The second assumption is related to the fact that the FMN molecule is released from the protein to solvent due to oxidative modifications of the protein. The FMN released to water leads to an increase of ${ }^{1} \mathrm{O}_{2}$ production outside of the protein. The presence of FMN in the aqueous environment was also observed in the work of Westberg et al. ${ }^{23}$ at low irradiation. In this work, we found evidence that FMN is present in the aqueous environment and its amount in the solution increases with irradiation of the sample.

Determination of ${ }^{3} \mathrm{FMN}$ lifetime. The time-resolved ${ }^{3} \mathrm{FMN}$ absorbance at $633 \mathrm{~nm}$ of the AsLOV2 C450A and the AsLOV2 wt are shown in insets of Fig. 1. The obtained ${ }^{3} \mathrm{FMN}$ lifetime values for both AsLOV2 variants are summarized in Table 1. The black points belong to low irradiation (obtained during the first $12.5 \mathrm{~min}$ of the irradiation), while the data taken after extensive irradiation $(75 \mathrm{~min})$ of the samples are shown in magenta.

Singlet oxygen phosphorescence. The experimental time-courses of the ${ }^{1} \mathrm{O}_{2}$ phosphorescence signals are shown in Fig. 1A,B for the AsLOV2 C450A and the AsLOV2 wt, respectively. The phosphorescence kinetics changes dramatically upon prolonged irradiation of the samples. For both AsLOV2 variants, the intensity of the phosphorescence signal increased significantly after $75 \mathrm{~min}$ of irradiation, indicating enhanced ${ }^{1} \mathrm{O}_{2}$ production. Analysis of the ${ }^{1} \mathrm{O}_{2}$ phosphorescence signals relies on the ${ }^{3} \mathrm{FMN}$ lifetime values, $\tau_{\mathrm{T}, \mathrm{i}}$, determined in the flash photolysis experiments. For all the six irradiation levels, the time-dependence of singlet oxygen phosphorescence $P(t)$ was assumed to consist of independent contributions, which correspond to different ${ }^{3}$ FMN groups: 


\begin{tabular}{|l|l|l|l|l|}
\hline \multirow{2}{*}{} & \multicolumn{3}{|l|}{ AsLOV2 C450A } & AsLOV2 wt \\
\cline { 2 - 5 } & $\begin{array}{l}\text { low irradiation } \\
(\mathbf{1 2 . 5} \text { min })\end{array}$ & $\begin{array}{l}\text { high irradiation } \\
(\mathbf{7 5} \text { min })\end{array}$ & $\begin{array}{l}\text { low irradiation } \\
(\mathbf{1 2 . 5} \text { min })\end{array}$ & $\begin{array}{l}\text { high irradiation } \\
(\mathbf{7 5} \text { min })\end{array}$ \\
\hline$\tau_{\mathrm{T}}^{\text {prot }}[\mu \mathrm{s}]$ & $57 \pm 2$ & $1.5 \pm 0.01$ & \multicolumn{2}{l|}{} \\
\hline$\tau_{\mathrm{T}}{ }^{\text {prot* }}[\mu \mathrm{s}]$ & $32 \pm 7$ & $23 \pm 3$ & - & - \\
\hline$\tau_{\mathrm{T}}{ }^{\text {water }}[\mu \mathrm{s}]$ & $2.7^{\mathrm{F}}$ & $2.7^{\mathrm{F}}$ & \multicolumn{2}{l|}{} \\
\hline$\tau_{\Delta}[\mu \mathrm{s}]$ & $2.7 \pm 0.2$ & $3.0 \pm 0.2$ & $2.6 \pm 0.2$ & $3.3 \pm 0.2$ \\
\hline
\end{tabular}

Table 1. The lifetime values of ${ }^{3} \mathrm{FMN}$ and ${ }^{1} \mathrm{O}_{2}$ as determined by fitting the time-resolved absorbance and phosphorescence data. ${ }^{\mathrm{F}}$ fixed value, not varied during the fitting procedure.
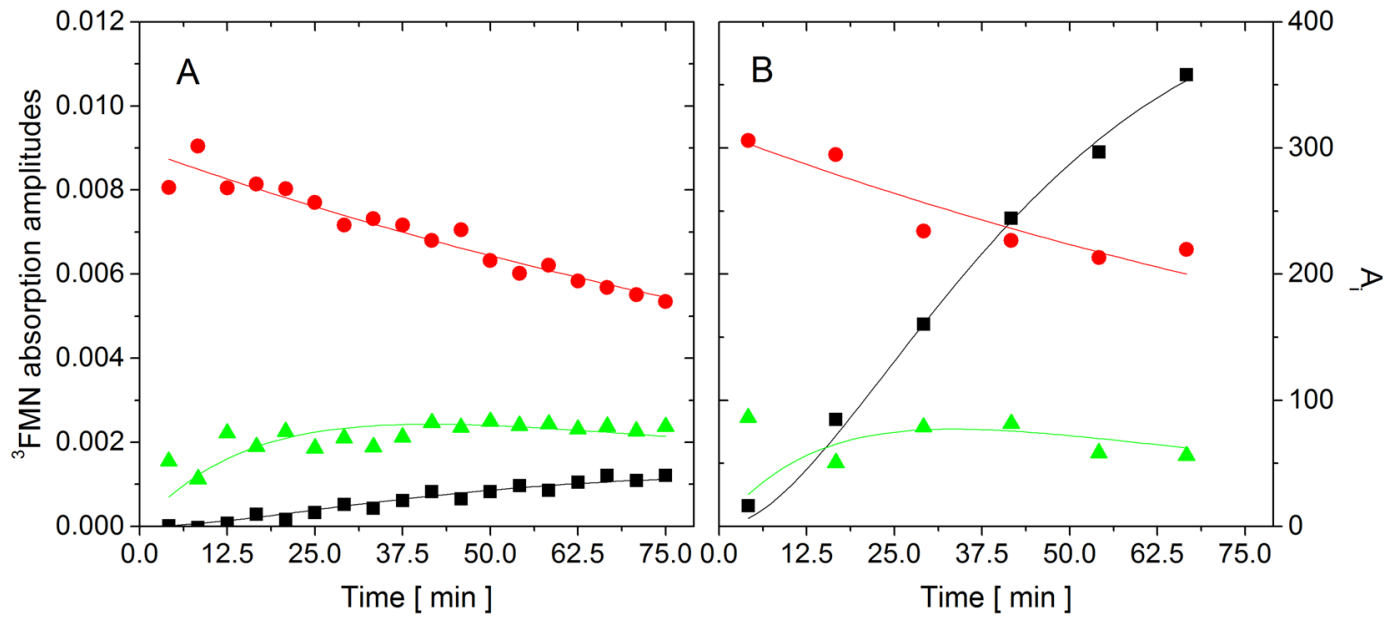

Figure 2. The amplitudes of the exponential ${ }^{3} \mathrm{FMN}$ absorption decays, $\mathrm{Abs}_{\mathrm{i}}{ }^{0}(\mathbf{A})$ and the amplitudes $\mathrm{A}_{\mathrm{i}}$ of the different ${ }^{3} \mathrm{FMN}$ group contributions to the ${ }^{1} \mathrm{O}_{2}$ phosphorescence signal (see Eq. 1) (B) in intact protein (red circles), in oxidized protein (green triangles), and in water (black squares) as a function of the irradiation time of AsLOV2 C450A. The solid lines represent the results of the fitting procedures.

$$
P(t)=\sum_{i}\left[\frac{A_{i} \tau_{\Delta}}{\tau_{\Delta}-\tau_{\mathrm{T}, \mathrm{i}}}\left(e^{-\frac{t}{\tau_{\Delta}}}-e^{-\frac{t}{\tau_{\mathrm{T}, \mathrm{i}}}}\right)\right]
$$

where $A_{\mathrm{i}}$ is amplitude of the phosphorescence and $\tau_{\Delta}$ is the lifetime of the ${ }^{1} \mathrm{O}_{2}$ molecule.

In agreement with the assumption that singlet oxygen, wherever being produced, spends most of its lifetime in the aqueous environment, only a single lifetime of singlet oxygen, $\tau_{\Delta}$, was assigned to all the different contributions. It is noted that this approximation holds true due to the relatively short singlet oxygen lifetime in water environment and low protein concentration.

In the following sections, detailed analyses of ${ }^{3} \mathrm{FMN}$ lifetimes and ${ }^{1} \mathrm{O}_{2}$ phosphorescence are presented for the AsLOV2 C450A and AsLOV2 wt.

AsLOV2 C450A. The ${ }^{3} \mathrm{FMN}$ lifetime. Based on the transient absorption signals measured with the AsLOV2 C450A, we concluded that three different FMN groups are present in the system. These groups were assigned to the FMN inside the intact protein, the FMN inside the oxidized protein and the FMN in the water environment. The corresponding ${ }^{3} \mathrm{FMN}$ lifetimes $\left(\tau_{\mathrm{T}}^{\text {prot }}, \tau_{\mathrm{T}}^{\text {prot*}}\right.$, and $\left.\tau_{\mathrm{T}}{ }^{\text {water }}\right)$ were determined by fitting all the 18 decay curves with triple-exponential decays using the following constrictions: $\tau_{\mathrm{T}}^{\text {prot }}$ - a single fitting parameter used for all the decay curves; $\tau_{\mathrm{T}}^{\text {prot* }}$ - no restrictions, this lifetime was allowed to evolve during the irradiation; $\tau_{\mathrm{T}}{ }^{\text {water }}$ - not fitted, fixed to $2.7 \mu \mathrm{s}$ - the lifetime measured by our apparatus in aqueous solution of FMN, which is in agreement with previously reported value ${ }^{2}$.

The ${ }^{3} \mathrm{FMN}$ lifetime values obtained for AsLOV2 C450A can be summarized as follows: $\tau_{\mathrm{T}}^{\text {prot }}=57 \pm 2 \mu$ s; $\tau_{\mathrm{T}}^{\text {prot* }}$ - gradually decreases from $32 \pm 7 \mu \mathrm{s}$ (at low-irradiation) to $23 \pm 3 \mu$ s (at high-irradiation) (Figure S1). The decreasing $\tau_{\mathrm{T}}^{\text {prot* }}$ lifetime is likely caused by the gradually increasing accessibility of the protein interior to oxygen due to by the irradiation-induced oxidation. The ${ }^{3} \mathrm{FMN}$ lifetime determined for AsLOV2 C450A, $\tau_{\mathrm{T}}^{\text {prot }}=57 \mu \mathrm{s}$, is in accordance with the corresponding lifetime values previously reported for the C450A variant, i.e. $72 \mu s^{31}$ and $98 \mu \mathrm{s}^{47}$, and also with the lifetimes found in miniSOG, $\tau_{\mathrm{T}}^{\text {prot }}=28 \mu \mathrm{s}$, and SOPP, $\tau_{\mathrm{T}}^{\text {prot }}=79 \mu \mathrm{s}$ at $30^{\circ} \mathrm{C}^{26}$.

Additional information can be derived from the amplitudes $\mathrm{Abs}_{\mathrm{i}}{ }^{0}$ of the triple-exponential decay curves $\left(\sum_{\mathrm{i}} \mathrm{Abs}_{\mathrm{i}}{ }^{0} \exp \left(-\mathrm{t} / \tau_{\mathrm{Ti}}\right)\right)$, which represents ${ }^{3} \mathrm{FMN}$ absorption right after the excitation laser pulse (Fig. $\left.2 \mathrm{~A}\right)$. The direct comparison of the initial absorption amplitudes is difficult because of the unknown extinction coefficients for all three different ${ }^{3} \mathrm{FMN}$ groups. Despite this drawback, the absorption amplitudes provide an important information about the kinetics of the system evolution. 


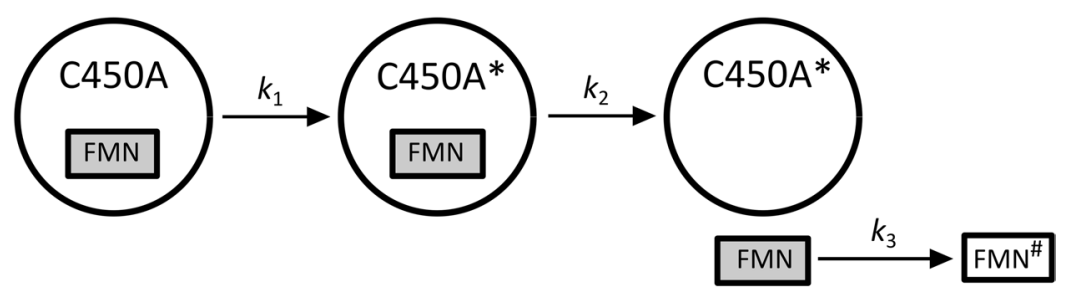

Scheme 1. The scheme of the irradiation-induced changes in AsLOV2 C450A and FMN dissociation. C450A and $\mathrm{C}_{450 \mathrm{~A}}$ * represent the intact and the oxidized protein, respectively. FMN and FMN ${ }^{\#}$ represent the intact and the bleached flavin cofactor, respectively.

Based on the experimental observation, we proposed a model that schematically describes irradiation-induced changes in AsLOV2 C450A accompanied by FMN release (Scheme 1).

The protein oxidation is characterized by the unimolecular rate constant $k_{1}$. The constant $k_{2}$ represents the rate constant of the FMN release from the oxidized protein. For the sake of simplicity, only a single unimolecular rate constant $k_{2}$ was used for all the proteins with different level of oxidation. Finally, the bleaching of FMN in the water environment (rate constant $k_{3}$ ) was taken into account in the model. Precise description of the model by differential equations and the corresponding analytical solutions for different FMN groups' concentrations $[\mathrm{FMN}]_{\mathrm{i}}$ are shown in Supporting information. It is noted that the values of $k_{1}, k_{2}$, and $k_{3}$ depend on the irradiation conditions and are specific for the present experiment.

To determine the rate constant of FMN bleaching, $k_{3}$, in the solution, we performed separate experiments. For this purpose, $25 \mu \mathrm{M}$ FMN was mixed with $25 \mu \mathrm{M}$ apoAsLOV2, which was prepared according to the procedure of Dürr et al. ${ }^{48}$ and was irradiated in the same way as both AsLOV2 variants. Noteworthy, upon admixture of apoAsLOV2 and FMN, formation of holoAsLOV2 was not detected based on UV-Vis absorbance measurement. The apoAsLOV2 wt was used to mimic the presence of the protein in the solution. As expected, significant bleaching of FMN was observed and both the production of singlet oxygen and the initial absorbance values $\mathrm{Abs}^{0}{ }_{\text {water }}$ of ${ }^{3} \mathrm{FMN}$ decreased exponentially. The rate of the bleaching was determined as $1 / k_{3}=3260 \mathrm{~s}$, which reflects both the decay of ${ }^{1} \mathrm{O}_{2}$ production, and the decay of the ${ }^{3} \mathrm{FMN}$ absorption amplitudes $\left(\mathrm{Abs}^{0}{ }_{\text {water }}\right)$. The ${ }^{3} \mathrm{FMN}$ lifetime remained unaffected, $2.7 \pm 0.1 \mu \mathrm{s}$, during the irradiation experiment.

The obtained kinetics of ${ }^{3} \mathrm{FMN}$ absorption amplitudes (Fig. 2A) for AsLOV2 C450A were fitted according to the model shown in Scheme 1 (assuming proportionality between: (i) the concentration of FMN, (ii) the amount of ${ }^{3} \mathrm{FMN}$ produced and (iii) the ${ }^{3} \mathrm{FMN}$ absorption signal $\mathrm{Abs}_{\mathrm{i}}{ }^{0}$ in each group, throughout the irradiation experiment). The data points of $\mathrm{Abs}^{0}{ }_{\text {prot }}$ were fitted by the single exponential decay (Eq. S1), from which the characteristic time, $1 / k_{1}=9000 \mathrm{~s}$ was obtained for the rate of protein oxidation in our system. The value of $k_{2}$ was determined by fitting the ${ }^{3} \mathrm{FMN}$ absorption in the oxidized protein $\mathrm{Abs}^{0}{ }_{\text {prot* }}^{*}$ and in the solution $\mathrm{Abs}^{0}{ }_{\text {water }}$ with the corresponding time-dependences (Eqs. S2 and S3), using the previous values of $k_{1}$ and $k_{3}$. The best match of the experimental data and the analytical curves was obtained for $1 / k_{2}=1000 \mathrm{~s}$. Based on the fits shown in Fig. $2 \mathrm{~A}$, we can conclude that the experimental data are well described by the model.

AsLOV2 C450A. Singlet oxygen phosphorescence. The ${ }^{3} \mathrm{FMN}$ lifetime values $\left(\tau_{\mathrm{T}}^{\text {prot }}, \tau_{\mathrm{T}}^{\text {prot* }}\right.$, and $\left.\tau_{\mathrm{T}}{ }^{\text {water }}\right)$ were utilized to analyze the ${ }^{1} \mathrm{O}_{2}$ phosphorescence data. The phosphorescence amplitudes $\mathrm{A}_{\text {prot }}, \mathrm{A}_{\text {prot* }}$ and $\mathrm{A}_{\text {water }}$ and the lifetime of singlet oxygen $\tau_{\Delta}$ were determined in a global fit (using all the measured curves shown in Fig. 1A). The lifetime of ${ }^{1} \mathrm{O}_{2}$ was allowed to evolve during the irradiation. The fitted curves and the obtained amplitudes are shown in Figs. $1 \mathrm{~A}$ and $2 \mathrm{~B}$, respectively. The evolutions of $\mathrm{A}_{\text {water }}$ and $\mathrm{A}_{\text {prot }}$ were fitted with the corresponding time-dependences of $[\mathrm{FMN}]_{\text {water }}$ and $[\mathrm{FMN}]_{\text {prot. }}$, respectively. The general shape of these curves reproduces the experimental data very well. Based on the proposed model, the proportionality factors of the two curves (black and red line in Fig. 2B) can be used to calculate the quantum yield of singlet oxygen production in the intact AsLOV2 C450A, using the quantum yield of ${ }^{1} \mathrm{O}_{2}$ production by FMN in the solution $\left(\Phi_{\Delta \text { water }}=0.57\right)^{2}$ as a reference. Taking into account the different absorbance of the two FMN groups at the excitation wavelength $(475 \mathrm{~nm})$ (Figure S2), the $\Phi_{\Delta}$ value for the intact AsLOV2 C450A equals to 0.07. The analogous quantitative analysis of the $\mathrm{A}_{\text {prot* }}$ data is not feasible due to the changing level of oxidation during the experiment.

AsLOV2 wt. The ${ }^{3} \mathrm{FMN}$ lifetime. The kinetics of ${ }^{3} \mathrm{FMN}$ transient absorption in the AsLOV2 wt (inset Fig. 1B), were analyzed in analogous way as for the AsLOV2 C450A variant. In the case of the AsLOV2 wt, only two triplet state lifetime components were identified. Based on this observation, we concluded that FMN is released from the oxidized AsLOV2 wt very fast. This is in agreement with our results from molecular dynamics studies, which indicate a steric clash between the oxidized Cys 450 and FMN (see below). The ${ }^{3} \mathrm{FMN}$ absorption decays were fitted with two lifetime values $\left(\tau_{\mathrm{T}}^{\text {prot }}, \tau_{\mathrm{T}}{ }^{\text {water }}\right)$, assuming that these lifetimes do not change during the irradiation: $\tau_{\mathrm{T}}^{\text {prot }}$ - a single fitting parameter used for all the decay curves; $\tau_{\mathrm{T}}^{\text {water }}$ - not fitted, fixed to $2.7 \mu \mathrm{s}$, which is the lifetime measured in pure FMN solution. The global fit resulted in a value of $\tau_{\mathrm{T}}^{\text {prot }}=1.50 \pm 0.01 \mu \mathrm{s}$. The low error indicates high-quality fits.

The obtained lifetime of the ${ }^{3} \mathrm{FMN}$ state in AsLOV2 wt is in good agreement with the results of Swartz et al., $\tau_{\mathrm{T}}{ }^{\text {prot }}=2 \mu \mathrm{s}^{31}$ and Song et al., $\tau_{\mathrm{T}}^{\text {prot }}=2.2 \mu \mathrm{s}^{49}$, but differs from the triplet state decay time reported by Gil et al., $\tau_{\mathrm{T}}{ }^{\text {prot }}=9.5 \mu \mathrm{s}^{47}$. 


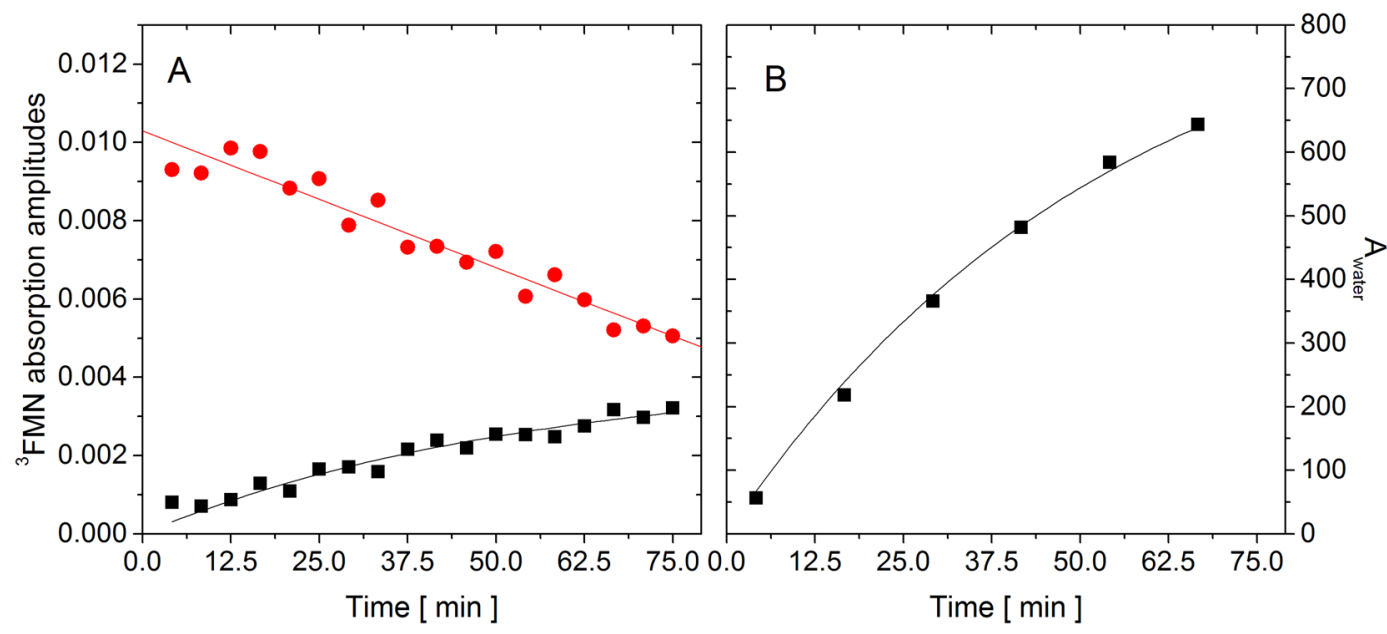

Figure 3. The amplitudes of the exponential ${ }^{3} \mathrm{FMN}$ absorption decays, $\mathrm{Abs}_{\mathrm{i}}{ }^{0}(\mathbf{A})$ and the amplitudes $\mathrm{A}_{\mathrm{i}}$ of the different ${ }^{3} \mathrm{FMN}$ group contributions to the ${ }^{1} \mathrm{O}_{2}$ phosphorescence signal (see Eq. 1) (B) in intact protein (red circles) and in water (black squares) as a function of the irradiation time of AsLOV2 wt. The solid lines represent the results of the fitting procedures.

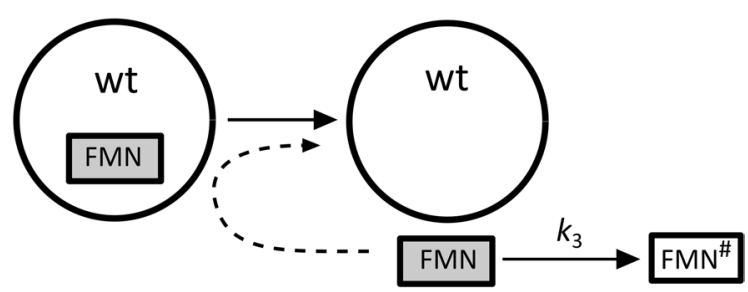

Scheme 2. The scheme of the irradiation-induced changes in AsLOV2 wt and FMN dissociation. FMN and $\mathrm{FMN}^{\#}$ represent the intact and the bleached flavin cofactor, respectively.

The amplitude of the ${ }^{3} \mathrm{FMN}$ absorption $\mathrm{Abs}^{0}{ }_{\text {prot }}$ indicates that the amount of ${ }^{3} \mathrm{FMN}$ produced in the intact protein (Fig. 3A, red points) decreased by $50 \%$. At the same time, there is no evidence of slowing the decrease down towards longer irradiations. In this case, the experimental data are better fitted with a linear decrease than with an exponential decay. This behavior is consistent with the assumption of the fast FMN release from the protein and can be rationalized as follows: the quantum yield of ${ }^{1} \mathrm{O}_{2}$ production is very low for the FMN inside the AsLOV2 wt. Once the FMN is released from the protein, the rate of ${ }^{1} \mathrm{O}_{2}$ production increases, which in turn enhances the rate of the protein oxidation and the rate of FMN release. In principle, the FMN release acts as an auto-catalyzed reaction, which explains the unusual (quasi-linear) decrease of $\mathrm{Abs}^{0}{ }_{\text {prot }}$. Scheme 2 shows a model of irradiation-induced changes in the AsLOV wt accompanied by FMN release.

The mathematical description of this model is shown in the Supporting information. This model was used to fit the $\mathrm{Abs}_{\mathrm{i}}{ }^{0}$ values in Fig. $3 \mathrm{~A}$. The curvature of the $\mathrm{Abs}_{\text {water }}$ (black squares), reflecting the bleaching effect, is well described by the rate constant $k_{3}$.

AsLOV2 wt. Singlet oxygen phosphorescence. The procedure of the analysis of ${ }^{1} \mathrm{O}_{2}$ phosphorescence data of the AsLOV2 wt were analogous as in the case of AsLOV2 C450A, taking into account the different model of irradiation-induced changes in the protein. Based on the fitting results, we conclude that the observed phosphorescence signal in AsLOV2 wt can be fully explained by ${ }^{1} \mathrm{O}_{2}$ production in the solution and the contribution of the $\mathrm{FMN}_{\text {prot }}$ group can be considered as negligible. The results of the phosphorescence global fits and the obtained amplitudes $A_{\text {water }}$ are shown in Figs. $1 \mathrm{~B}$ and $3 \mathrm{~B}$, respectively. The $\mathrm{A}_{\text {water }}$ values are well fitted with the $[\mathrm{FMN}]_{\text {water }}$ time-dependence using the same value of $1 / k_{3}=3260 \mathrm{~s}$.

The global fit (based on the developed model) allowed us to determine the dependence of ${ }^{1} \mathrm{O}_{2}$ lifetime on irradiation time (Fig. 4). The results show than in both AsLOV2 variants the lifetime of ${ }^{1} \mathrm{O}_{2}$ increases with irradiation time very likely as a result of progressive proteins oxidation.

Changes in AsLOV2 optical spectra induced by irradiation. UV-VIS absorption spectroscopy. UV-VIS absorption spectra of the wild type and the C450A variant of AsLOV2 are almost identical in the spectral range $300-550 \mathrm{~nm}$ and correspond to the absorption of FMN (Figure S2). Both variants exhibit major absorption peaks at $447 \mathrm{~nm}, 473 \mathrm{~nm}$, and a shoulder at $\sim 425 \mathrm{~nm}$. The difference between these two forms in the region 300-400 $\mathrm{nm}$ reflects the replacement of cysteine for alanine in position $450^{31,32}$. After blue light irradiation, a small spectral change in the region $400-500 \mathrm{~nm}$ is observed in both AsLOV2 forms, corresponding to $12 \%$ and 


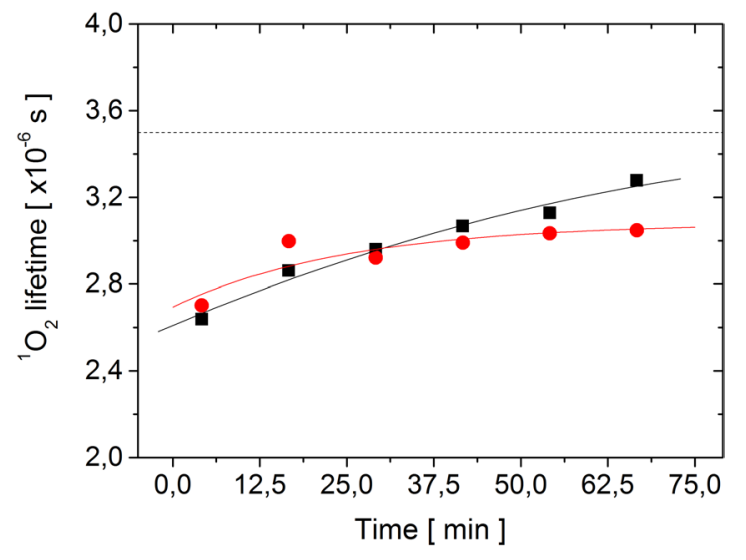

Figure 4. Dependence of the ${ }^{1} \mathrm{O}_{2}$ lifetime on irradiation time in AsLOV2 C450A (red circles) and AsLOV2 wt (black square). The estimated error of individual data points is $\pm 0.2 \mu \mathrm{s}$. The solid lines serves as an eye lead. The dashed line is the ${ }^{1} \mathrm{O}_{2}$ lifetime in pure water ${ }^{70}$.

$\sim 22 \%$ decrease in the absorbance at $447 \mathrm{~nm}$ for AsLOV2 C450A and AsLOV2 wt, respectively (Figure S3). The observed decrease is usually attributed to chromophore bleaching. The small increase in the absorbance in the region 300-400 $\mathrm{nm}$ has been previously interpreted as a result of tryptophan oxygenation to kynurenine $\mathrm{e}^{50}$, which is in accordance with the results obtained by mass spectrometry (see below).

Circular dichroism. Structural changes in AsLOV2 proteins induced by irradiation were also followed by circular dichroism (CD) spectroscopy in the far- and near- UV regions (Figure S4). In the far-UV region, the CD spectra show that both variants of AsLOV2 contain similar fractions of $\alpha$-helical $(\sim 13 \pm 3 \%)$ and $\beta$-sheet $(32 \pm 3 \%)$ structures (for the analysis we used two different web servers: DICHROWEB ${ }^{51,52}$ and BeStSel ${ }^{53}$ ). Small changes in the secondary structure in both variants of AsLOV2 are noticeable after the extensive laser irradiation (Figures S4A,C), corresponding to $\sim 13 \%$ and $\sim 25 \%$ decrease in ellipticity at $222 \mathrm{~nm}$ for AsLOV2 C450A and AsLOV2 wt, respectively. The extent of the changes is similar both in the far-UV and the near-UV regions to those observed in the corresponding UV-VIS absorption spectra. The observed changes can be attributed to a direct effect of ROS or can be of a secondary nature as a result of the chromophore oxygenation and/or its release ${ }^{54}$.

Fluorescence. AsLOV2 contains two intrinsic fluorescent probes, FMN chromophore and one tryptophan residue, Trp491. Comparison of the fluorescence emission spectra of the flavin chromophore located in the AsLOV2 wt and its variant AsLOV2 C450A clearly shows significantly lower intensity of the fluorescence in the AsLOV2 wt (Fig. 5). This is due to light-induced formation of covalent bond between flavin chromophore and the reactive cysteine in the AsLOV2 wt. The fluorescence maximum of the wild-type protein after irradiation is moved to higher wavelengths, corresponding to the peak of free FMN at $520 \mathrm{~nm}$ (Fig. 5A). These results suggest that part of the flavin chromophores are released from the binding pocket into the solvent. In fact, we fitted the fluorescence spectrum of AsLOV2 wt after irradiation as a combination of the fluorescence spectra of protein bound FMN and free FMN. The obtained fit consists of two fractions combining $\sim 45 \%$ of the free FMN and $\sim 55 \%$ protein-bound FMN (Fig. 5A). In the case of AsLOV2 C450A, the irradiation induces $~ 23 \%$ decrease in fluorescence intensity measured at $500 \mathrm{~nm}$ (Fig. 5B). An analogous fit for AsLOV2 C450A (Fig. 5B) led to fractions of free and protein-bound FMN equal to $\sim 25 \%$ and $\sim 75 \%$, respectively. On the other hand, the intrinsic tryptophan fluorescence of AsLOV2 C450A before irradiation is $\sim 20 \%$ higher than the fluorescence of AsLOV2 wt (insets Fig. 5). The irradiation induces $\sim 1.6$-fold and $\sim 2.6$-fold increase in fluorescence intensity of AsLOV2 C450A and AsLOV2 wt, respectively. The absence of a shift in the maximum of the tryptophan fluorescence suggests that the observed irradiation-induced increase is not due to a conformational change in the proteins, but rather due to decreased fluorescence quenching by the flavin cofactor.

To address the possibility of FMN dissociation from the protein, we performed simple filtration experiments in which the released cofactor passes through the filter, while the protein is retained. The outcome of these experiments showed that before irradiation there was no free FMN detected in the filtrate. The irradiation-induced changes in the proteins led to release of FMN from both types of proteins (Fig. 6). Although this method does not allow quantitative determination of released FMN, it does allow a relative comparison: irradiation induced a release of $\sim 1.8$-fold more FMN in the case AsLOV2 wt in comparison with AsLOV2 C450A variant (Fig. 6), which corresponds to the ratio of dissociated FMN from AsLOV2 wt and AsLOV2 C450A obtained from the analysis of the fluorescence spectra in Fig. 5.

Another way to assess the fraction of retained FMN in the protein after irradiation is to compare the amplitude of the cysteinyl-FMN adduct formation in the AsLOV2 wt measured by flash-induced absorbance changes at $447 \mathrm{~nm}^{55}$ using samples before and after the extensive $(75 \mathrm{~min}$ ) irradiation by laser (Fig. 7). The obtained results suggest that after irradiation only $\sim 40 \%$ of FMN is able to form the adduct.

Determination of irradiation-induced changes in primary structures of AsLOV2. Light-induced production of ${ }^{1} \mathrm{O}_{2}$ by FMN is accompanied by covalent modification of amino acids in AsLOV2, which 


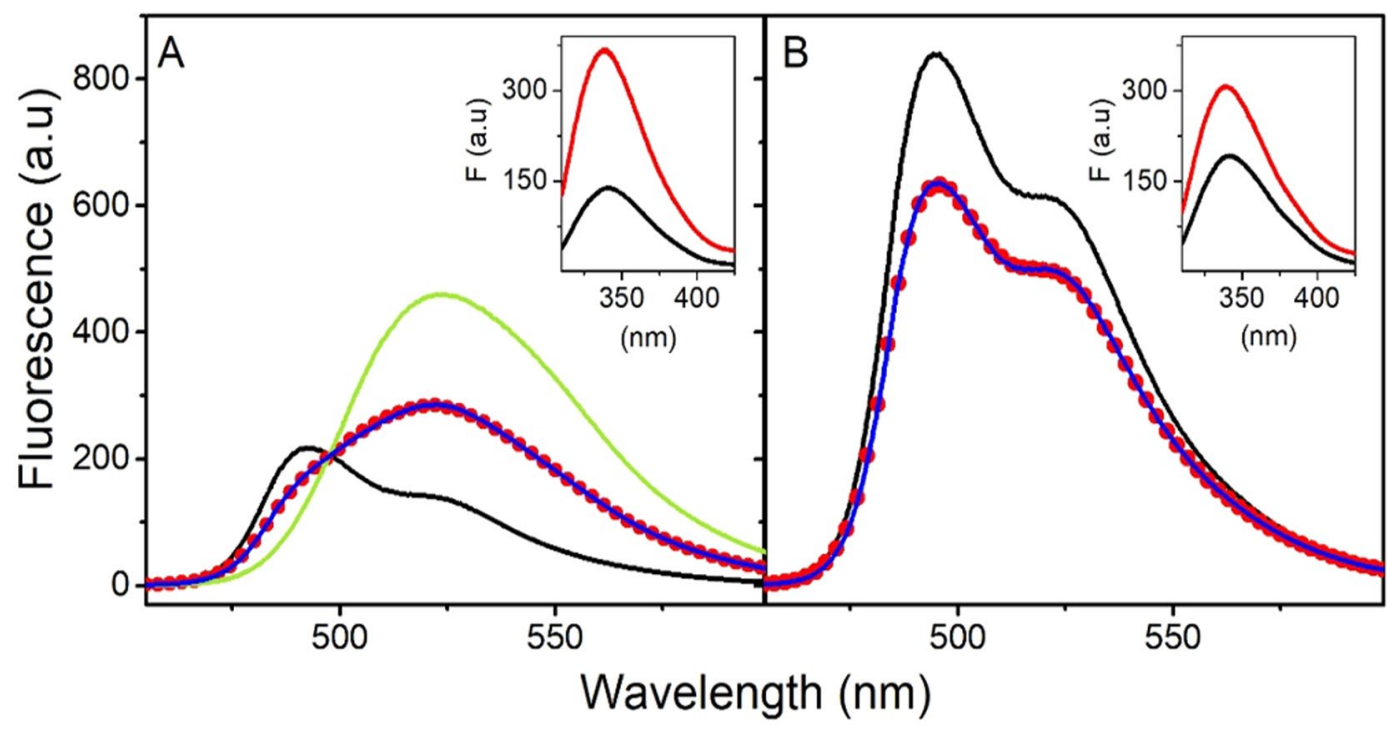

Figure 5. Fluorescence emission spectra of FMN and intrinsic tryptophan in AsLOV2 wt and AsLOV C450A. The fluorescence of FMN in AsLOV2 wt (A) and in AsLOV2 C450A (B) before (black line) and after (red dots) illumination and the fluorescence of free FMN (green). The blue line shows the fit to FMN fluorescence after the illumination. The fits (blue line) consist of two fractions of fluorescence: protein-bound and free FMN. Insets: Intrinsic tryptophan fluorescence of the corresponding forms of AsLOV2 before (black line) and after illumination (red line). The concentrations of proteins and the free FMN were $10 \mu \mathrm{M}$ in $20 \mathrm{mM}$ Tris- $\mathrm{HCl}, \mathrm{pH} 7.8$.

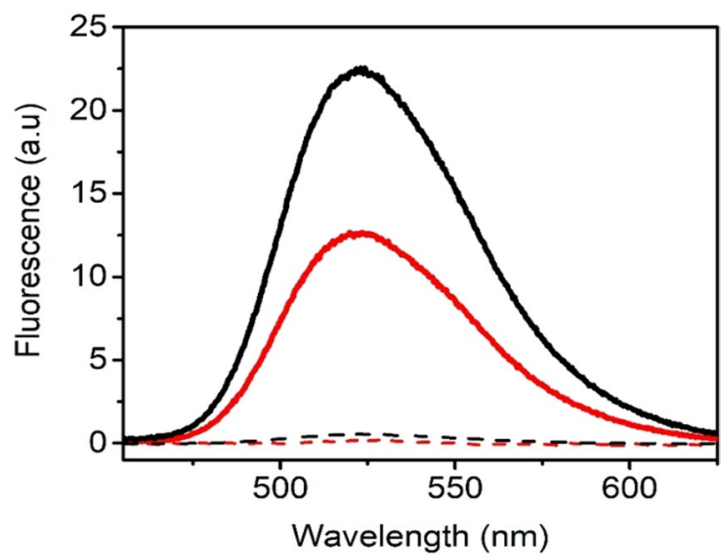

Figure 6. Analysis of a release of FMN from AsLOV2 wt and AsLOV2 C450A induced by blue light irradiation. FMN fluorescence indicates a release of FMN from its binding pocket to the bulk solvent. FMN fluorescence of non-irradiated AsLOV2 wt (black dashed), AsLOV2 C450A (red dashed) and irradiated AsLOV2 wt (black solid) and AsLOV2 C450A (red solid).

localization and nature of the modification were determined. First, we measured the intact mass to verify the protein state and possible fragmentation. Based on these data (Figure S5A), we concluded that the irradiated proteins are intact, no significant fragmentation occurred but they differ in the extent of oxidative modifications, with C450A form being significantly more affected upon irradiation (Figure S5B).

In the bottom-up approach, the protein was digested in solution, analyzed by LC-MS/MS and the generated peptides were identified by database searching and automated de novo sequencing. This yielded a list of modified residues and allowed a direct comparison between the different AsLOV2 states regarding the extent of oxidation of individual amino acids (Fig. 8). By this approach, 97\% coverage was achieved and only five residues (460-464) were missed (Figure S6). Observed oxidation of the His-tag sequence (N-terminal Met and histidines) are not listed among the modified residues in Fig. 8, as this part of the sequence (the first 31 amino acids) is unnatural for AsLOV2 (Figure S6).

In the AsLOV2 wt, di- and tri- oxidation of Cys450, (but not single oxidation) were found to be very prominent modifications. While the bottom-up method targeted the whole bulk of protein populations, the top-down approach allowed the selection of the "first-hit"/singly oxidized species, and their subsequent fragmentation and assignment of modified residues based on the protein MS/MS spectra. The selection of singly oxidized protein forms had also another benefit as it "filtered-out" the Cys450 modification, which occurred as double and triple 


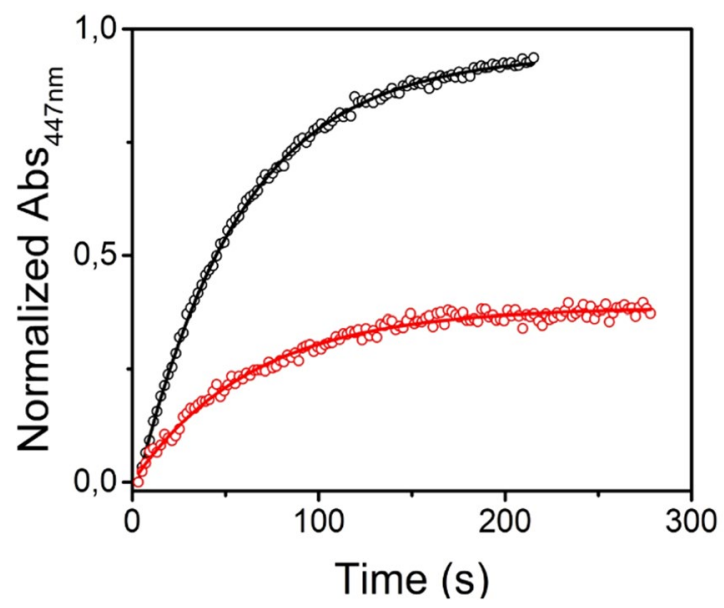

Figure 7. Dark state recovery kinetics of non-irradiated (black) and irradiated (red) AsLOV2 wt. Experimental data are shown as circles and the solid lines show fits of the data by single exponential functions.

\begin{tabular}{|c|c|c|c|c|c|c|}
\hline & Mod. & wt-N [\%] & mut-N [\%] & wt-I [\%] & mut-1 [\%] $\left.\right|^{\mid}$ & $\begin{array}{l}\text { mut-I - mut-N } \\
\text { / wt-I - wt-N }\end{array}$ \\
\hline F415: & +01 & 0.0 & 0.0 & 2.8 & 3.8 & 1.4 \\
\hline P420 & $-\mathrm{C} 101$ & 0.0 & 0.0 & 19.3 & 19.8 & 1.0 \\
\hline P423/426 & $-\mathrm{C101}$ & 0.0 & 0.3 & 11.1 & 14.7 & 1.3 \\
\hline$F 429$ & +01 & 0.0 & 1.0 & 19.0 & 21.5 & 1.1 \\
\hline C450 & $+02 /+03$ & - & - & 82.5 & - & $*$ \\
\hline F452/P456 & +01 & 0.0 & 0.0 & 0.1 & 0.2 & 2.0 \\
\hline P456 & $-\mathrm{C101}$ & 0.0 & 0.0 & 0.3 & 1.0 & 3.0 \\
\hline Y483 & +01 & 0.0 & 0.0 & 3.5 & 3.7 & 1.1 \\
\hline F490/W491 & +01 & 1.1 & 1.6 & 18.4 & 16.3 & 0.9 \\
\hline W491 & $-\mathrm{C101}$ & 0.0 & 0.0 & 7.9 & 7.4 & 0.9 \\
\hline M499 & +01 & 10.0 & 9.8 & 29.5 & 44.0 & 1.8 \\
\hline Y508 & +01 & 0.0 & 0.2 & 1.2 & 1.0 & 0.7 \\
\hline M530 & +01 & 0.6 & 0.0 & 6.1 & 11.7 & 2.1 \\
\hline
\end{tabular}

Figure 8. List of oxidation products and their extent of oxidation (intensity of oxidatively modified peptide) in all studied protein forms - wild-type (wt) and C450A (mut) before (N) and after (I) irradiation. Column Mod shows the elemental composition of the modification (oxidation: $+\mathrm{O} 1,+\mathrm{O} 2$ or $+\mathrm{O} 3$; Trp to kynurenin: $-\mathrm{C} 1+\mathrm{O} 1$; Pro to pyrrolidone: $-\mathrm{C} 1 \mathrm{O} 1)$. When it was not possible to clearly assign oxidation to one specific residue, two possibly affected residues are listed in the first column. The last column highlights the fold change in oxidation between C450A and wt forms. In case of C450 oxidation (marked in the last column with an asterisk) such comparisons were not possible due to generation of different peptides upon oxidation and due to mutation. The intensity of oxidized form was indirectly deduced from intensity decrease of the intact, unoxidized Asp-N generated peptide.

oxidation and allowed us to focus on other residues. Unfortunately, the gas-phase fragmentation of AsLOV2 proved to be quite inefficient as we missed fragmentation in the middle of the protein (Figure S7A). Nonetheless, we obtained the information supporting and complementing our bottom-up data. Based on the oxidation increase between the N-terminal fragment ions b26 and b47 together with the same trend observed from the opposite site (C-terminal fragment ions y127 and y151) we can point on Phe 415 as an oxidation-sensitive amino acid. Furthermore, we can also conclude (based on fragment ion y36, Figure S7B), that the sequence spanning from Gly511 to the C-terminus is not affected by oxidative events in any of the analyzed AsLOV2 forms. This is at first in apparent contrast with the bottom-up data, where Met530 was found to be oxidized and where the data point to a significant difference between wt and C450A forms (Fig. 8). However, one should keep in mind that the top-down fragmentation was aimed at the first-hit oxidation and thus we can assume that Met530 oxidation is a secondary event. Finally, we can state that the remaining primary oxidation sites are localized between amino acids 420 and 511 where the majority of significantly oxidized residues were found by the bottom-up approach.

Altogether, based on the MS data it can be concluded that: (i) the modifications occurring in AsLOV2 upon irradiation consist of various products of amino acid side chain oxidations, (ii) the modifications are light-dependent as the overall extent of the oxidative modifications is much higher in irradiated samples of both 
A

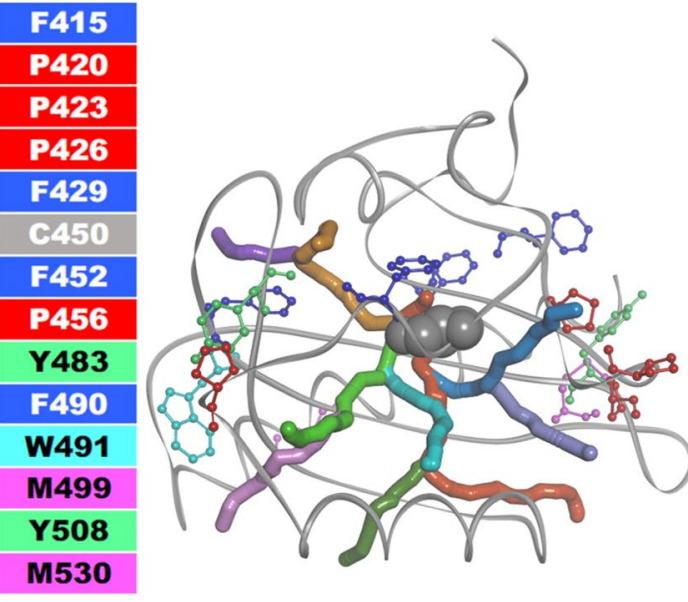

B

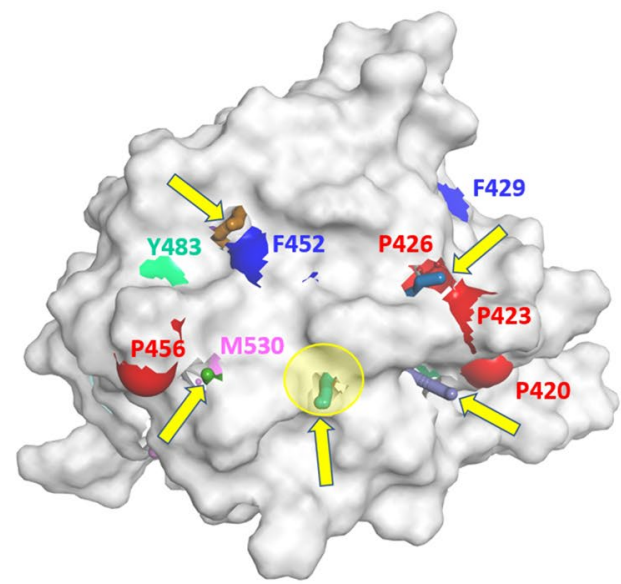

Figure 9. Discovery Studio visualization of Caver-calculated tunnels of minimized PDB ID: 2v0u structure. (A) Ribbon representation of the protein with color-coded amino acids (summarized in Fig. 6) shown in ball and stick representation. C450 is shown in grey CPK representation. The color coding of the tunnels represented as $\mathrm{CPK}$ does not correspond to the color coding of the amino acids and is for their distinction only. (B) Surface representation of the protein with color coding of the amino acids of interest on the surface. The yellow arrows highlight the tunnels reaching the protein surface. The ribityl chain of FMN structure (not shown) overlaps with the circled tunnel 1 (yellow semitransparent circle). Detailed information from $5 \mathrm{~ns}$ MD simulations is summarized in Figure S9.

AsLOV2 wt and the AsLOV2 C450A variant, (iii) generally the same amino acids are oxidized in both AsLOV2 variants, (iv) the extent of some oxidative modification is higher in AsLOV2 C450A compared to AsLOV2 wt.

Detection of tunnels in the structure of AsLOV2. The unusual modification of proline residues (highlighted in red in Figs. 9 and S8, illustrating that P420, P423 and P426 form a cluster on the right side of the protein) led us to an assumption that in this part of the protein exit channel(s) may exist, connecting the source of the production of ${ }^{1} \mathrm{O}_{2}$ with the protein surface. As a result, even prolines, which are usually resistant to redox reactions, are oxidized by reactive oxygen species created by FMN. Indeed, the Caver $3.0^{45}$ tunnel analysis of AsLOV2 wt crystal structure (PDB ID: 2v0u) detected the presence of several tunnels. We have analyzed several AsLOV2-related protein structures: at first we considered their optimized geometries and then their geometries resulting from $5 \mathrm{~ns}$ MD simulation in water environment: (i) dark state of AsLOV2 wt (PDB ID: 2v0u), i.e. without covalent bond between $\mathrm{C} 450$ and $\mathrm{C} 4$ (a) of the isoalloxazine ring (Figure S8A), (ii) in silico $\mathrm{SOO}^{-}$substitution on C450 Sulphur (Figure S8B), (iii) light state of AsLOV2 wt (PDB ID: 2v0w), i.e. with covalent bond between $\mathrm{C} 450$ and $\mathrm{C} 4(\mathrm{a})$ of the isoalloxazine ring (Figure S8C), and (iv) in silico mutated AsLOV2 C450A (Figure S8D). The presence of tunnels was detected in all the protein geometries (Figures S8A-D). However, the number of tunnel clusters, as indicated on the pie chart on Figure S8 slightly differs, related to the C450 modifications/FMN binding. Several tunnel clusters (channels) (Figs. 9 and S8) are believed to be lined by the above mentioned P420, P423 and P426 amino acids. Several other channels, as illustrated in Figs. 9 and S8 terminate in the proximity of the highlighted amino acids listed in Fig. 8 and are shown as CPK representation in Figure S8. Interestingly, the comparison of a number of channels observed in MD simulations in AsLOV2 wt and AsLOV2 C450A suggests that C450A exhibits slightly more tunnels and could thus suffer a larger damage by oxygen than AsLOV2 wt. The highest number of calculated tunnels was found in $2 \mathrm{v} 0 \mathrm{u}-\mathrm{SOO}^{-}$, which in the light of our results might point to increased dynamics of the protein matrix explaining thus more efficient release of FMN from the protein.

\section{Discussion}

The protein matrix surrounding FMN can efficiently decrease the $\Phi_{\Delta}$ value by quenching the excited triplet state of FMN and by restriction of oxygen diffusion towards the isoalloxazine ring ${ }^{56}$. This, on one side, may have a protective role for the photosensitizers close surrounding by preventing unwanted production of ROS but on the other hand, significantly limits the ROS effect on the site of an intendent action. Here, we argue that genetically-encoded photosensitizers may be used as a carrier of the reactive cofactor, and the release of the cofactor can be modulated by an irradiation or possibly other perturbation of the protein matrix.

Primary structure modification of AsLOV2 upon irradiation. As can be deduced from the fine structure of FMN absorption spectrum in the wavelength range $400-500 \mathrm{~nm}$, the cofactor has no extensive contacts with solvent molecules ${ }^{57}$. This is also in accordance with the crystal structures of the closely related flavoproteins SOPP and miniSOG ${ }^{2}$. On the other hand, our in silico analysis of the AsLOV2 crystal structure ${ }^{58}$ suggests the existence of several channels in the protein structure, connecting the reactive site $\mathrm{C}(4 \mathrm{a})$ on the isoalloxazine ring with the protein surface (Fig. 9). This connection becomes even more obvious after molecular dynamics simulation (Figure S8). The presence of channels was also identified by molecular dynamics 
simulation in the case of miniSOG ${ }^{59}$. If the channels play an active role as a traffic route of molecular oxygen to the FMN binding site and diffusion of the ${ }^{1} \mathrm{O}_{2}$ and possibly other ROS out to the protein surface, one can expect oxidatively modified amino acids localized close to these channels. Indeed, the localization of three modified prolines, Pro420, Pro423, and Pro426, at the orifice of a cluster of several channels provide an explanation of unexpected modification of this otherwise very oxidation-resistant amino acid (Fig. 9). Our results strongly support the ability of reactive oxygen species to react with proline $e^{60,61}$. Because some reports conclude that proline does not interact with ${ }^{1} \mathrm{O}_{2}$ our observations suggest a production of other ROS than ${ }^{1} \mathrm{O}_{2}$ by FMN when encapsulated in AsLOV2 ${ }^{62-64}$.

ROS effectively react with aromatic amino acids such as Trp and Tyr. One surprising observation regarding oxidative modification of amino acids in AsLOV2 was that none of the three tyrosine residues, present in AsLOV2, were modified by ROS produced by FMN. There are two ROS species that can be produced by FMN, $\mathrm{O}_{2}{ }^{\bullet-}$ and ${ }^{1} \mathrm{O}_{2}$. While $\mathrm{O}_{2}{ }^{\bullet-}$ is relatively unreactive and directly reacts only at a few specific protein sites ${ }^{65}$, ${ }^{1} \mathrm{O}_{2}$ reacts efficiently with five common and functionally important amino acids (Cys, His, Met, Trp, Tyr) ${ }^{5,8,66}$. However, our findings show that ROS produced by AsLOV2 variants, surprisingly, do not oxidize the tyrosine residues of AsLOV2. Noteworthy, the absence of modification of tyrosine residues by ${ }^{1} \mathrm{O}_{2}$ was also noticed in the case of two unrelated proteins such as cytochrome $c$ (containing 4 Tyr residues) ${ }^{67}$ and lysozyme (containing 3 Tyr residues $)^{68}$.

The absence of the irradiation-induced modification of electron rich amino acids such as Tyr and Phe in both variants of AsLOV2 is unexpected on chemical grounds. Kiselar et al. ${ }^{69}$ speculated that some of the oxidative modification of aromatic residues in general can be "transferred" to Met by radical transfer. This suggestion could help explain the rather significant increase in Met oxidation, but only low Tyr and Phe oxidation in C450A.

A higher number of oxidative modifications of amino acids detected in our work in comparison with a very recent paper by Torra et al. ${ }^{29}$ can be attributed to the differences in the solution irradiation (wavelength, power, etc.) and partially also to the higher sensitivity and resolution obtained by our LC-MS/MS analysis on FT-ICR MS and combination of proteomics approaches directed at localization of the modification sites. Our approach truly analyzes all protein states present in the sample and, in addition, the top-down analysis allows the identification of the first-hit residues. In contrast, Torra et al..$^{29}$ identified the oxidized residues in the crystal structure, hence the possibility that they observed just the crystallization-capable population cannot be excluded.

Approaches to increase singlet oxygen production by LOV domains. The models based on our data demonstrate the fact that irradiation of both AsLOV2 variants is accompanied by FMN dissociation from the protein matrix. However, the dissociation as well as the production of ${ }^{1} \mathrm{O}_{2}$ is more efficient in AsLOV2 wt than in AsLOV2 $\mathrm{C} 450 \mathrm{~A}$. The observed increase in ${ }^{1} \mathrm{O}_{2}$ yield is thus rather the consequence of FMN release than of the protein oxidation.

In several previous studies, the authors assumed that an increase in ${ }^{1} \mathrm{O}_{2}$ production was due to blocking concurrent reactions by electron transfer from redox-active amino acids such as Tyr, Phe, Met, Trp, and Cys, which quench the triplet state of FMN and may thus lead to formation of $\mathrm{O}_{2}{ }^{-29}$. In fact, this assumption led to efforts to rationally design genetically encoded efficient flavoproteins by replacing amino acids responsible for quenching of the FMN triplet state $e^{23,26}$, allowing thus increased production of ${ }^{1} \mathrm{O}_{2}$. These efforts indeed led to an increased $\Phi_{\Delta}$ value attained in miniSOG and SOPP without detecting a dissociation of $\mathrm{FMN}^{21,26}$. These observations suggest that ${ }^{1} \mathrm{O}_{2}$ production in genetically-encoded photosensitizers can be achieved also without a release of the cofactor from the protein matrix.

Impact of mutation C450A on FMN release. The combined analyses of the data obtained from ${ }^{3} \mathrm{FMN}$ absorption experiments (Fig. 3A), from the analytical models as well as from fitting of irradiation-induced FMN fluorescence of the free and protein-bound FMN (Fig. 5) point to the release of approximately $48 \pm 3 \%$ and $28 \pm 4 \%$ of FMN from AsLOV2 wt and AsLOV2 C450A, respectively, after irradiation of the proteins. About 1.8-fold higher release of FMN from AsLOV2 wt in comparison with AsLOV2 C450A was documented by direct determination of relative amount of FMN released from the irradiated proteins (Fig. 6). All these results suggest that AsLOV2 wt is more affected by irradiation than its variant C450A. Strikingly, the amplitude of irradiation-induced changes in absorbance and ellipticity (Figures S3 and S4) as well as the extent of changes detected by mass spectrometry analysis (Figure S7) clearly show the opposite, i.e. that the variant C450A is more perturbed than AsLOV2 wt. In fact, the analysis of the total (integrated) ${ }^{1} \mathrm{O}_{2}$ phosphorence signal in both variants clearly shows that AsLOV C450A produced significantly higher amount of ${ }^{1} \mathrm{O}_{2}$ (Figure S10) and likely also other ROS during irradiation, which explains the higher oxidation damage of AsLOV2 C450A in our experiments.

To reconcile our observations, we hypothesize that more efficient release of FMN from AsLOV2 wt than from its Ala-containing variant is due to irradiation-induced oxidation of Cys450. As illustrated in Figure S9, the "brute force" superposition of C450 and (per)oxidized C450 results in a steric clash of modified Cys450 and FMN. Molecular geometry optimization and the subsequent MD simulations can easily eliminate such inappropriate molecular contacts, but in real structures such intermolecular conflicts could facilitate the release of FMN from AsLOV2.

\section{Conclusions}

We show that an irradiation-induced increase of ${ }^{1} \mathrm{O}_{2}$ production in the AsLOV2 variants is due to a release of FMN to solvent as a result of oxidative modification of certain amino acids, predominantly the reactive cysteine 450 , localized nearby the isoalloxazine ring in the AsLOV2 structure. Our findings may be utilized to design more efficient genetically encoded photosensitizers based on LOV domains. The protein scaffold can serve merely as a targetable carrier while the reactive cofactor would be released at the site of action by a suitable perturbation of the protein structure. In principle, intensive blue light irradiation or combined approach including both 
irradiation and thermogenesis could be applied. The irradiation can be more efficient in releasing FMN, if the binding site of isoalloxazine ring becomes repulsive upon irradiation, either through steric clashes or through charge repulsion. Enhanced effect, might be achieved by placing suitable amino acids close to the isoalloxazine ring. These amino acids, such as cysteine or methionine, upon irradiation-induced oxidation increase their volumes and form steric clashes, thereby repelling the flavin cofactor. Local thermogenesis could be an alternative approach that would increase FMN dissociation from AsLOV2, due to the increased dynamics of polypeptide chain and consequently the increase of the $\Phi_{\Delta}$ value $^{2}$.

Received: 13 September 2019; Accepted: 13 February 2020;

Published online: 05 March 2020

\section{References}

1. Baier, J. et al. Singlet oxygen generation by UVA light exposure of endogenous photosensitizers. Biophys. J. 91, 1452-1459, https:// doi.org/10.1529/biophysj.106.082388 (2006).

2. Westberg, M., Bregnhoj, M., Etzerodt, M. \& Ogilby, P. R. Temperature Sensitive Singlet Oxygen Photosensitization by LOV-Derived Fluorescent Flavoproteins. The journal of physical chemistry. B 121, 2561-2574, https://doi.org/10.1021/acs.jpcb.7b00561 (2017).

3. Baron, R. et al. Multiple pathways guide oxygen diffusion into flavoenzyme active sites. Proceedings of the National Academy of Sciences of the United States of America 106, 10603-10608, https://doi.org/10.1073/pnas.0903809106 (2009).

4. Meissner, B., Schleicher, E., Weber, S. \& Essen, L. O. The dodecin from Thermus thermophilus, a bifunctional cofactor storage protein. The Journal of biological chemistry 282, 33142-33154, https://doi.org/10.1074/jbc.M704951200 (2007).

5. Davies, M. J. Singlet oxygen-mediated damage to proteins and its consequences. Biochem. Biophys. Res. Commun. 305, 761-770, https://doi.org/10.1016/s0006-291x(03)00817-9 (2003).

6. Ogilby, P. R. Singlet oxygen: there is indeed something new under the sun. Chem. Soc. Rev. 39, 3181-3209, https://doi.org/10.1039/ b926014p (2010).

7. Schweitzer, C. \& Schmidt, R. Physical mechanisms of generation and deactivation of singlet oxygen. Chem Rev 103, 1685-1757, https://doi.org/10.1021/cr010371d (2003).

8. Davies, M. J. Reactive species formed on proteins exposed to singlet oxygen. Photochemical \& photobiological sciences: Official journal of the European Photochemistry Association and the European Society for Photobiology 3, 17-25, https://doi.org/10.1039/ b307576c (2004).

9. Mansoori, B. et al. Photodynamic therapy for cancer: role of natural products. Photodiagnosis Photodyn Ther 26, 395-404, https:// doi.org/10.1016/j.pdpdt.2019.04.033 (2019).

10. McLean, M. A. et al. Mechanism of chromophore assisted laser inactivation employing fluorescent proteins. Anal. Chem. 81, 1755-1761, https://doi.org/10.1021/ac801663y (2009).

11. Riani, Y. D., Matsuda, T., Takemoto, K. \& Nagai, T. Green monomeric photosensitizing fluorescent protein for photo-inducible protein inactivation and cell ablation. BMC Biol. 16, 50, https://doi.org/10.1186/s12915-018-0514-7 (2018).

12. Redmond, R. W. \& Kochevar, I. E. Spatially resolved cellular responses to singlet oxygen. Photochem Photobiio 82, 1178-1186, https://doi.org/10.1562/2006-04-14-IR-874 (2006).

13. Wojtovich, A. P. \& Foster, T. H. Optogenetic control of ROS production. Redox Biol 2, 368-376, https://doi.org/10.1016/j. redox.2014.01.019(2014).

14. Westberg, M. et al. Exerting better control and specificity with singlet oxygen experiments in live mammalian cells. Methods 109, 81-91, https://doi.org/10.1016/j.ymeth.2016.07.001 (2016).

15. Endres, S. et al. An optogenetic toolbox of LOV-based photosensitizers for light-driven killing of bacteria. Sci Rep 8, 15021, https:// doi.org/10.1038/s41598-018-33291-4 (2018).

16. Hilgers, F. et al. Genetically Encoded Photosensitizers as Light-Triggered Antimicrobial Agents. Int J Mol Sci 20, 4608, https://doi. org/10.3390/ijms20184608 (2019).

17. Shirmanova, M. et al. Towards PDT with Genetically Encoded Photosensitizer KillerRed: A Comparison of Continuous and Pulsed Laser Regimens in an Animal Tumor Model. PLoS ONE 10, e0144617, https://doi.org/10.1371/journal.pone.0144617 (2015).

18. Norman, R. A. Past and future: porphyria and porphyrins. Skinmed 4, 287-292, https://doi.org/10.1111/j.1540-9740.2005.03706.x (2005).

19. Xiong, Y., Tian, X. \& Ai, H. W. Molecular Tools to Generate Reactive Oxygen Species in Biological Systems. Bioconjug Chem 30, 1297-1303, https://doi.org/10.1021/acs.bioconjchem.9b00191 (2019).

20. Jiang, H. N., Li, Y. \& Cui, Z. J. Photodynamic Physiology-Photonanomanipulations in Cellular Physiology with Protein Photosensitizers. Front Physiol 8, 191, https://doi.org/10.3389/fphys.2017.00191 (2017).

21. Ruiz-Gonzalez, R. et al. Singlet oxygen generation by the genetically encoded tag miniSOG. J. Am. Chem. Soc. 135, 9564-9567, https://doi.org/10.1021/ja4020524 (2013).

22. Rodriguez-Pulido, A. et al. Correction: Assessing the potential of photosensitizing flavoproteins as tags for correlative microscopy. Chem Commun (Camb) 52, 9300, https://doi.org/10.1039/c6cc90313d (2016).

23. Westberg, M., Bregnhoj, M., Etzerodt, M. \& Ogilby, P. R. No Photon Wasted: An Efficient and Selective Singlet Oxygen Photosensitizing Protein. The journal of physical chemistry. B 121, 9366-9371, https://doi.org/10.1021/acs.jpcb.7b07831 (2017).

24. Jimenez-Banzo, A. et al. Singlet oxygen photosensitisation by GFP mutants: oxygen accessibility to the chromophore. Photochemical \& photobiological sciences: Official journal of the European Photochemistry Association and the European Society for Photobiology 9 , 1336-1341, https://doi.org/10.1039/c0pp00125b (2010).

25. Ragas, X., Cooper, L. P., White, J. H., Nonell, S. \& Flors, C. Quantification of photosensitized singlet oxygen production by a fluorescent protein. ChemPhysChem 12, 161-165, https://doi.org/10.1002/cphc.201000919 (2011).

26. Westberg, M., Holmegaard, L., Pimenta, F. M., Etzerodt, M. \& Ogilby, P. R. Rational design of an efficient, genetically encodable, protein-encased singlet oxygen photosensitizer. J. Am. Chem. Soc. 137, 1632-1642, https://doi.org/10.1021/ja511940j (2015).

27. Shu, X. et al. A genetically encoded tag for correlated light and electron microscopy of intact cells, tissues, and organisms. PLoS biology 9, e1001041, https://doi.org/10.1371/journal.pbio.1001041 (2011).

28. Jensen, R. L., Arnbjerg, J. \& Ogilby, P. R. Reaction of singlet oxygen with tryptophan in proteins: a pronounced effect of the local environment on the reaction rate. J. Am. Chem. Soc. 134, 9820-9826, https://doi.org/10.1021/ja303710m (2012).

29. Torra, J. et al. Tailing miniSOG: structural bases of the complex photophysics of a flavin-binding singlet oxygen photosensitizing protein. Sci Rep 9, 2428, https://doi.org/10.1038/s41598-019-38955-3 (2019).

30. Zayner, J. P., Antoniou, C. \& Sosnick, T. R. The amino-terminal helix modulates light-activated conformational changes in AsLOV2. J. Mol. Biol. 419, 61-74, https://doi.org/10.1016/j.jmb.2012.02.037 (2012).

31. Swartz, T. E. et al. The photocycle of a flavin-binding domain of the blue light photoreceptor phototropin. The Journal of biological chemistry 276, 36493-36500, https://doi.org/10.1074/jbc.M103114200 (2001).

32. Salomon, M., Christie, J. M., Knieb, E., Lempert, U. \& Briggs, W. R. Photochemical and mutational analysis of the FMN-binding domains of the plant blue light receptor, phototropin. Biochemistry 39, 9401-9410 (2000). 
33. Desmond Molecular Dynamics System. Schrödinger Maestro 2019-2; Desmond Molecular Dynamics System; Schrödinger Inc. \& D. E. Shaw Research; New York, NY (2019).

34. Bowers, K. J. et al. in Proceedings of the 2006 ACM/IEEE conference on Supercomputing - SC '06 (ACM Press, 2006).

35. Jorgensen, W. L. \& Tirado-Rives, J. The OPLS [optimized potentials for liquid simulations] potential functions for proteins, energy minimizations for crystals of cyclic peptides and crambin. J. Am. Chem. Soc. 110, 1657-1666, https://doi.org/10.1021/ja00214a001 (1988).

36. Jorgensen, W. L., Maxwell, D. S. \& TiradoRives, J. Development and testing of the OPLS all-atom force field on conformational energetics and properties of organic liquids. J. Am. Chem. Soc. 118, 11225-11236, https://doi.org/10.1021/Ja9621760 (1996).

37. Damm, W., Frontera, A., TiradoRives, J. \& Jorgensen, W. L. OPLS all-atom force field for carbohydrates. J. Comput. Chem. 18, 1955-1970, 10.1002/(Sici)1096-987x(199712)18:16<1955::Aid-Jcc1>3.3.Co;2-A (1997).

38. McDonald, N. A. \& Jorgensen, W. L. Development of an all-atom force field for heterocycles. Properties of liquid pyrrole, furan, diazoles, and oxazoles. J. Phys. Chem. B 102, 8049-8059, https://doi.org/10.1021/Jp981200o (1998).

39. Jorgensen, W. L. \& McDonald, N. A. Development of an all-atom force field for heterocycles. Properties of liquid pyridine and diazenes. Theochem-Journal of Molecular Structure 424, 145-155, https://doi.org/10.1016/s0166-1280(97)00237-6 (1998).

40. Rizzo, R. C. \& Jorgensen, W. L. OPLS All-Atom Model for Amines: Resolution of the Amine Hydration Problem. J. Am. Chem. Soc. 121, 4827-4836, https://doi.org/10.1021/ja984106u (1999).

41. Kaminski, G. A., Friesner, R. A., Tirado-Rives, J. \& Jorgensen, W. L. Evaluation and Reparametrization of the OPLS-AA Force Field for Proteins via Comparison with Accurate Quantum Chemical Calculations on Peptides $\dagger$. The Journal of Physical Chemistry B 105, 6474-6487, https://doi.org/10.1021/jp003919d (2001).

42. Watkins, E. K. \& Jorgensen, W. L. Perfluoroalkanes: Conformational Analysis and Liquid-State Properties from ab Initio and Monte Carlo Calculations. The Journal of Physical Chemistry A 105, 4118-4125, https://doi.org/10.1021/jp004071w (2001).

43. Berendsen, H. J. C., Grigera, J. R. \& Straatsma, T. P. The Missing Term in Effective Pair Potentials. J. Phys. Chem.-Us 91, 6269-6271, https://doi.org/10.1021/J100308a038 (1987).

44. Humphrey, W., Dalke, A. \& Schulten, K. VMD: visual molecular dynamics. J. Mol. Graph. 14(33-38), 27-38 (1996).

45. Chovancova, E. et al. CAVER 3.0: a tool for the analysis of transport pathways in dynamic protein structures. PLoS Comput. Biol. 8 , e1002708, https://doi.org/10.1371/journal.pcbi.1002708 (2012).

46. Dassault Systemes BIOVIA; Discovery Studio Client; San Diego, USA. Dassault Systemes BIOVIA; Discovery Studio 2019 Client; San Diego, USA (2019)

47. Gil, A. A. et al. Femtosecond to Millisecond Dynamics of Light Induced Allostery in the Avena sativa LOV Domain. The journal of physical chemistry. B 121, 1010-1019, https://doi.org/10.1021/acs.jpcb.7b00088 (2017).

48. Durr, H., Salomon, M. \& Rudiger, W. Chromophore exchange in the LOV2 domain of the plant photoreceptor phototropin 1 from oat. Biochemistry 44, 3050-3055, https://doi.org/10.1021/bi0478897 (2005).

49. Song, S. H. et al. Modulating LOV domain photodynamics with a residue alteration outside the chromophore binding site. Biochemistry 50, 2411-2423, https://doi.org/10.1021/bi200198x (2011).

50. Fukunaga, Y., Katsuragi, Y., Izumi, T. \& Sakiyama, F. Fluorescence characteristics of kynurenine and N'-formylkynurenine. Their use as reporters of the environment of tryptophan 62 in hen egg-white lysozyme. Journal of biochemistry 92, 129-141, https://doi. org/10.1093/oxfordjournals.jbchem.a133909 (1982).

51. Lobley, A., Whitmore, L. \& Wallace, B. A. DICHROWEB: an interactive website for the analysis of protein secondary structure from circular dichroism spectra. Bioinformatics 18, 211-212 (2002).

52. Sreerama, N. \& Woody, R. W. Estimation of protein secondary structure from circular dichroism spectra: comparison of CONTIN, SELCON, and CDSSTR methods with an expanded reference set. Anal. Biochem. 287, 252-260, https://doi.org/10.1006/ abio.2000.4880 (2000).

53. Micsonai, A. et al. BeStSel: a web server for accurate protein secondary structure prediction and fold recognition from the circular dichroism spectra. Nucleic Acids Res. 46, W315-W322, https://doi.org/10.1093/nar/gky497 (2018).

54. Tomasková, N., Varinska, L. \& Sedlak, E. Rate of oxidative modification of cytochrome $\mathrm{c}$ by hydrogen peroxide is modulated by Hofmeister anions. General Physiology and Biophysics 29, 255-265, https://doi.org/10.4149/gpb_2010_03_255 (2010).

55. Zayner, J. P. \& Sosnick, T. R. Factors that control the chemistry of the LOV domain photocycle. PLoS ONE 9, e87074, https://doi. org/10.1371/journal.pone.0087074 (2014)

56. Leferink, N. G. et al. Identification of a gatekeeper residue that prevents dehydrogenases from acting as oxidases. The Journal of biological chemistry 284, 4392-4397, https://doi.org/10.1074/jbc.M808202200 (2009).

57. Yagi, K., Ohishi, N., Nishimoto, K., Choi, J. D. \& Song, P. S. Effect of hydrogen bonding on electronic spectra and reactivity of flavins. Biochemistry 19, 1553-1557, https://doi.org/10.1021/bi00549a003 (1980).

58. Halavaty, A. S. \& Moffat, K. N- and C-terminal flanking regions modulate light-induced signal transduction in the LOV2 domain of the blue light sensor phototropin 1 from Avena sativa. Biochemistry 46, 14001-14009, https://doi.org/10.1021/bi701543e (2007).

59. Pietra, F. Molecular dynamics simulation of dioxygen pathways through mini singlet oxygen generator (miniSOG), a genetically encoded marker and killer protein. Chem. Biodivers. 11, 1883-1891, https://doi.org/10.1002/cbdv.201400125 (2014).

60. Alia, A., Mohanty, P. \& Matysik, J. Effect of proline on the production of singlet oxygen. Amino Acids 21, 195-200, https://doi. org/10.1007/s007260170026 (2001).

61. Matysik, J., Alia, A., Bhalu, B. \& Mohanty, P. Molecular mechanisms of quenching of reactive oxygen species by proline under stress in plants. Current Science 82, 525-532 (2002).

62. Signorelli, S., Arellano, J. B., Melo, T. B., Borsani, O. \& Monza, J. Proline does not quench singlet oxygen: evidence to reconsider its protective role in plants. Plant physiology and biochemistry: PPB 64, 80-83, https://doi.org/10.1016/j.plaphy.2012.12.017 (2013).

63. Pimenta, F. M., Jensen, R. L., Breitenbach, T., Etzerodt, M. \& Ogilby, P. R. Oxygen-dependent photochemistry and photophysics of "miniSOG", a protein-encased flavin. Photochemistry and Photobiology 89, 1116-1126, https://doi.org/10.1111/php.12111 (2013).

64. Barnett, M. E., Baran, T. M., Foster, T. H. \& Wojtovich, A. P. Quantification of light-induced miniSOG superoxide production using the selective marker, 2-hydroxyethidium. Free radical biology \&. medicine 116, 134-140, https://doi.org/10.1016/j. freeradbiomed.2018.01.014 (2018).

65. Winterbourn, C. C. Reconciling the chemistry and biology of reactive oxygen species. Nat. Chem. Biol. 4, 278-286, https://doi. org/10.1038/nchembio.85 (2008).

66. Di Mascio, P. et al. Singlet Molecular Oxygen Reactions with Nucleic Acids, Lipids, and Proteins. Chem Rev 119, 2043-2086, https:// doi.org/10.1021/acs.chemrev.8b00554 (2019).

67. Kim, J. et al. Oxidative modification of cytochrome c by singlet oxygen. Free radical biology \& medicine 44, 1700-1711, https://doi. org/10.1016/j.freeradbiomed.2007.12.031 (2008).

68. Marques, E. F., Medeiros, M. H. G. \& Di Mascio, P. Lysozyme oxidation by singlet molecular oxygen: Peptide characterization using [(18) O]-labeling oxygen and nLC-MS/MS. Journal of mass spectrometry: JMS 52, 739-751, https://doi.org/10.1002/jms.3983 (2017).

69. Kiselar, J. G., Maleknia, S. D., Sullivan, M., Downard, K. M. \& Chance, M. R. Hydroxyl radical probe of protein surfaces using synchrotron X-ray radiolysis and mass spectrometry. International journal of radiation biology 78, 101-114, https://doi. org/10.1080/09553000110094805 (2002).

70. Jensen, R. L., Arnbjerg, J. \& Ogilby, P. R. Temperature effects on the solvent-dependent deactivation of singlet oxygen. J. Am. Chem. Soc. 132, 8098-8105, https://doi.org/10.1021/ja101753n (2010). 


\title{
Acknowledgements
}

This work was supported by the research grants provided by Slovak research and development agency (APVV-150069 and APVV-15-0485) and by the research grant VEGA 1/0557/20. Instrument access for mass spectrometry analysis was enabled through EU_FT-ICR_MS network funded by the EU Horizon 2020 grant 731077. IMIC group acknowledges support from CSF 19-16084S and infrastructure funding CZ.1.05/1.1.00/02.0109, LQ1604 and LM2015043 CIISB. Dr. Anna Kozarova (University of Windsor, Windsor, Ontario, Canada) is acknowledged for critical reading of the manuscript. This work forms a part of PhD study of M.P.

\section{Author contributions}

M.P., P.Mi., D.J., P.N., P.Ma., G.B. and E.S. designed the research, M.P., F.F., A.H., G.Y., T.K., M.-S.S. and G.B. performed experiments and analyzed data, P.Ma., A.P., D.J., G.B. and E.S. wrote the manuscript.

\section{Competing interests}

The authors declare no competing interests.

\section{Additional information}

Supplementary information is available for this paper at https://doi.org/10.1038/s41598-020-60861-2.

Correspondence and requests for materials should be addressed to G.B. or E.S.

Reprints and permissions information is available at www.nature.com/reprints.

Publisher's note Springer Nature remains neutral with regard to jurisdictional claims in published maps and institutional affiliations.

\begin{abstract}
(c) (1) Open Access This article is licensed under a Creative Commons Attribution 4.0 International License, which permits use, sharing, adaptation, distribution and reproduction in any medium or format, as long as you give appropriate credit to the original author(s) and the source, provide a link to the Creative Commons license, and indicate if changes were made. The images or other third party material in this article are included in the article's Creative Commons license, unless indicated otherwise in a credit line to the material. If material is not included in the article's Creative Commons license and your intended use is not permitted by statutory regulation or exceeds the permitted use, you will need to obtain permission directly from the copyright holder. To view a copy of this license, visit http://creativecommons.org/licenses/by/4.0/.
\end{abstract}

(C) The Author(s) 2020 